JOURNAL OF THE

AMERICAN MATHEMATICAL SOCIETY

Volume 19, Number 4, October 2006, Pages 759-779

S 0894-0347(06)00523-6

Article electronically published on March 17, 2006

\title{
DIVISIBILITY OF THE STABLE MILLER-MORITA-MUMFORD CLASSES
}

\author{
SOREN GALATIUS, IB MADSEN, AND ULRIKE TILLMANN
}

\section{INTRODUCTION AND RESULTS}

Let $\Gamma_{g, b}^{s}$ denote the mapping class group of a surface of genus $g$ with $b$ ordered boundary components and $s$ marked points. We will suppress $s$ or $b$ when their value is zero. Gluing a disk or a torus with two boundary components to one of the boundary components induces homomorphisms

$$
\Gamma_{g, b-1}^{s} \longleftarrow \Gamma_{g, b}^{s} \longrightarrow \Gamma_{g+1, b}^{s}
$$

Recall that by Harer-Ivanov's stability theory both homomorphisms induce a homology isomorphism in dimensions $*$ with $2 *+1<g$; cf. [H2], [I]. Let $\Gamma_{\infty}:=$ $\lim _{g \rightarrow \infty} \Gamma_{g, 2}$ be the stable mapping class group.

Mumford in $[\mathrm{Mu}$. introduced certain tautological classes in the cohomology of moduli spaces of Riemann surfaces. Miller [Mi] and Morita [Mo] studied topological analogues. Let $e \in H^{2}\left(B \Gamma_{g, b}^{1} ; \mathbb{Z}\right)$ be the Euler class of the central extension

$$
\mathbb{Z} \longrightarrow \Gamma_{g, b+1} \longrightarrow \Gamma_{g, b}^{1}
$$

which is induced by gluing a disk with a marked point to one of the boundary components. Define

$$
\kappa_{i}:=\pi_{!}\left(e^{i+1}\right) \in H^{2 i}\left(B \Gamma_{g, b} ; \mathbb{Z}\right)
$$

where $\pi_{\text {! }}$ is the Umkehr (or integration along the fibre) map associated to the forgetful map $\Gamma_{g, b}^{1} \rightarrow \Gamma_{g, b}$. These correspond under the maps of (1.1) when $i>0$ and hence define classes in $H^{*}\left(B \Gamma_{\infty} ; \mathbb{Z}\right)$. We will only be concerned with these stable classes in this paper.

By the proof of the Mumford conjecture [MW],

$$
H^{*}\left(B \Gamma_{\infty} ; \mathbb{Q}\right) \simeq \mathbb{Q}\left[\kappa_{1}, \kappa_{2}, \ldots\right]
$$

In contrast, little is known about $\kappa_{i}$ in integral cohomology though it follows from [H1] that $\kappa_{1}$ is precisely divisible by 12 (cf. [MT, p. 537]). We write

$$
H_{\text {free }}^{*}\left(B \Gamma_{\infty}\right):=H^{*}\left(B \Gamma_{\infty} ; \mathbb{Z}\right) / \text { Torsion }
$$

for the integral lattice in $H^{*}\left(B \Gamma_{\infty} ; \mathbb{Q}\right)$.

Received by the editors October 31, 2004.

2000 Mathematics Subject Classification. Primary 57R20, 55P47.

Key words and phrases. Mapping class group, characteristic classes, surface bundles.

The third author was supported by an Advanced Fellowship of the EPSRC.

(C)2006 American Mathematical Society Reverts to public domain 28 years from publication 
Theorem 1.1. Let $D_{i}$ be the maximal divisor of $\kappa_{i}$ in $H_{\text {free }}^{*}\left(B \Gamma_{\infty}\right)$. Then for all $i \geq 1$,

$$
D_{2 i}=2 \quad \text { and } \quad D_{2 i-1}=\operatorname{den}\left(\frac{B_{i}}{2 i}\right) .
$$

Here $B_{i}$ denotes the $i$-th Bernoulli number and den is the function that takes a rational number when expressed as a fraction in its lowest terms to its denominator. It is well known, cf. [MSt, Appendix B], that $\operatorname{den}\left(B_{i}\right)$ is the product of all primes $p$ such that $p-1$ divides $2 i$, and that a prime divides $\operatorname{den}\left(B_{i} / 2 i\right)$ if and only if it divides $\operatorname{den}\left(B_{i}\right)$. So in terms of their $p$-adic valuation the $D_{i}$ are determined by the formula

$$
\nu_{p}\left(D_{i}\right)=\left\{\begin{array}{lll}
1+\nu_{p}(i+1) & \text { if } i+1 \equiv 0 & \bmod (p-1) \\
0 & \text { if } i+1 \not \equiv 0 & \bmod (p-1),
\end{array}\right.
$$

and $D_{1}=2^{2} \cdot 3, D_{3}=2^{3} \cdot 3 \cdot 5, D_{5}=2^{2} \cdot 3^{2} \cdot 7, \ldots$.

Our Theorem 1.1 is inspired by a conjecture of T. Akita $\mathrm{Ak}$ which we also prove:

Theorem 1.2. The element $\kappa_{i}$ in $H^{2 i}\left(B \Gamma_{\infty} ; \mathbb{F}_{p}\right)$ vanishes if and only if $i+1 \equiv$ $0 \bmod (p-1)$.

Remark 1.3. The divisor $D_{i}$ of $\kappa_{i}$ in $H_{\text {free }}^{*}\left(B \Gamma_{\infty}\right)$ is not necessarily equal to the maximal divisor $D_{i}^{\mathbb{Z}}$ of $\kappa_{i}$ in integral cohomology $H^{*}\left(B \Gamma_{\infty} ; \mathbb{Z}\right)$ but only provides an upper bound for it. However, Theorem 1.2 gives

$$
p \text { divides } D_{i}^{\mathbb{Z}} \Longleftrightarrow p \text { divides } D_{i} \text {, }
$$

which was strengthened by the first author in G2 to

$$
p^{2} \text { divides } D_{i}^{\mathbb{Z}} \Longleftrightarrow p^{2} \text { divides } D_{i} \text {. }
$$

It follows that for all even $i$ and for many odd $i(i=1,5,9,13, \ldots), D_{i}$ is indeed equal to $D_{i}^{\mathbb{Z}}$, and one may expect that $D_{i}=D_{i}^{\mathbb{Z}}$ for all $i \geq 1$.

Remark 1.4. The integral lattice $H_{\text {free }}^{*}\left(B \Gamma_{\infty}\right)$ inherits a Hopf algebra structure. The graded module of primitive elements $P\left(H_{\text {free }}^{*}\left(B \Gamma_{\infty}\right)\right)$ is a copy of $\mathbb{Z}$ in each even degree, and $\kappa_{i}$ is a primitive element of $H_{\text {free }}^{*}\left(B \Gamma_{\infty}\right)$. The structure of the Hopf algebra $H_{\text {free }}^{*}\left(B \Gamma_{\infty}\right)$ is not completely understood at present, but we have the following partial results.

Theorem 1.5. For odd primes $p$ there is an isomorphism of Hopf algebras over the p-local integers $\mathbb{Z}_{(p)}$ :

$$
H_{\text {free }}^{*}\left(B \Gamma_{\infty} ; \mathbb{Z}_{(p)}\right) \simeq H^{*}\left(B U ; \mathbb{Z}_{(p)}\right) .
$$

This fails for $p=2$. In fact, the squaring map

$$
\xi: H_{\text {free }}^{2}\left(B \Gamma_{\infty}\right) \otimes \mathbb{F}_{2} \rightarrow H_{\text {free }}^{4}\left(B \Gamma_{\infty}\right) \otimes \mathbb{F}_{2}
$$

is not injective, so the algebra $H_{\text {free }}^{*}\left(B \Gamma_{\infty} ; \mathbb{Z}_{(2)}\right)$ is not polynomial.

In outline, the proofs of the above theorems depend on previous results as follows. For Theorem 1.2, the proof of the "if" part in Section 3.3 is a calculation of characteristic classes which relies on the fact that there is a map of infinite loop spaces $\alpha: \mathbb{Z} \times B \Gamma_{\infty}^{+} \rightarrow \Omega^{\infty} \mathbb{C} P_{-1}^{\infty}$ (compare [T] and [MT] or Theorems 2.1 and 2.2 below). The "only if" part is implied by Theorem 1.1: if $p$ divides $\kappa_{i}$, then in particular it must divide its reduction to the free part. 
For Theorem 1.1, we first establish a lower bound: $D_{2 i} \geq 2$ by the "if" part of Theorem 1.2 and $D_{2 i-1} \geq \operatorname{den}\left(B_{i} / 2 i\right)$ by a well-known relation between the $\kappa_{i}$ classes and the symplectic characteristic classes for surface bundles (here stated as Theorem 4.2). The main theorem of [MT provides an upper bound which is tight for $i$ even and precisely twice the lower bound for $i$ odd. To eliminate the indeterminacy of the factor 2, the main theorem of [MW] (Theorem 2.4 below), as well as calculations from [G1] (Proposition 4.4) and a stronger version of the main result of [MT] (given in Theorem 2.2 and proved in Section 5) are used.

Theorem 1.5 is proved in Section 6 .

Given the interest in the mapping class groups also outside the topology community we have strived to make this paper as self-contained as possible. In particular we have spelled out some of the more obscure parts of [MT].

\section{Spectrum COHOmology And EARlier RESUlts}

2.1. Spectra and spectrum cohomology. Let $E=\left\{E_{n}, \epsilon_{n}\right\}$ be a CW-spectrum 1 in the sense of [A1]: $E_{n}$ is a sequence of pointed CW-complexes and $\epsilon_{n}$ : $S E_{n} \rightarrow E_{n+1}$ a (pointed) isomorphism onto a subcomplex, where $S(-)$ denotes suspension. The associated infinite loop space is the direct limit

$$
\Omega^{\infty} E=\operatorname{colim} \Omega^{n} E_{n}
$$

of the $n$-th loop space of $E_{n}$; the limit is taken over the adjoint maps $\epsilon_{n}^{\prime}: E_{n} \rightarrow$ $\Omega E_{n+1}$.

The $k$-th homotopy group of $E$ is defined to be the direct limit of $\pi_{n+k}\left(E_{n}\right)$. It is equal to the $k$-th homotopy group of the space $\Omega^{\infty} E$. In particular, the group of components of $\Omega^{\infty} E$ is the direct limit of $\pi_{n}\left(E_{n}\right)$. For $\alpha \in \pi_{0}\left(\Omega^{\infty} E\right)$ we let $\Omega_{\alpha}^{\infty} E$ be the component determined by $\alpha$. In particular we write $\Omega_{0}^{\infty} E$ for the component of the zero element.

The homology and cohomology groups of $E$ are

$$
H^{k}(E)=\lim _{n \rightarrow \infty} \tilde{H}^{k+n}\left(E_{n}\right), H_{k}(E)=\operatorname{colim}_{n \rightarrow \infty} \tilde{H}_{k+n}\left(E_{n}\right),
$$

where the limits are induced from the maps $\epsilon_{n}$ together with the suspension isomorphisms 2 In contrast to homotopy groups the cohomology groups of a spectrum are usually much simpler than the cohomology groups of $\Omega^{\infty} E$.

The evident evaluation map from $S^{n} \Omega^{n} E_{n}$ to $E_{n}$ induces maps

$$
\sigma^{*}: H^{*}(E) \longrightarrow \tilde{H}^{*}\left(\Omega_{0}^{\infty} E\right), \sigma_{*}: \tilde{H}_{*}\left(\Omega_{0}^{\infty} E\right) \longrightarrow H_{*}(E) .
$$

If we use field coefficients in the cohomology groups, then $H^{*}\left(\Omega_{0}^{\infty} E\right)$ is a connected Hopf algebra and the image of $\sigma^{*}$ is contained in the graded vector space $P H^{*}\left(\Omega_{0}^{\infty} E\right)$ of primitive elements. We shall be particularly concerned with the torsion free integral homology and cohomology groups

$$
H_{\text {free }}^{*}\left(\Omega_{0}^{\infty} E\right)=H^{*}\left(\Omega_{0}^{\infty} E ; \mathbb{Z}\right) / \text { Torsion, } \quad H_{*}^{\text {free }}\left(\Omega_{0}^{\infty} E\right)=H_{*}\left(\Omega_{0}^{\infty} E ; \mathbb{Z}\right) / \text { Torsion. }
$$

\footnotetext{
${ }^{1}$ If one does not assume the spaces to be CW-complexes, then one should assume that $\epsilon_{n}$ is a closed cofibration.

${ }^{2}$ The $k$-th spectrum cohomology of $E$ is normally defined as the group of homotopy classes of degree $k$ spectrum maps from $E$ to the Eilenberg-Mac Lane spectrum $K(\mathbb{Z})$. This coincides with the above formula for $H^{k}(E)$ whenever for all $k$ the inverse system $\lim _{n \rightarrow \infty} \tilde{H}^{k+n}\left(E_{n}\right)$ satisfies the Mittag-Löffler condition (see A1]). In particular this will be the case when the structure maps $\epsilon: S E_{n} \rightarrow E_{n+1}$ are $c(n)$-connected for some function $c(n)$ with $c(n) \rightarrow \infty$ as $n \rightarrow \infty$.
} 
They are lattices in $H^{*}\left(\Omega_{0}^{\infty} E ; \mathbb{Q}\right)$ and $H_{*}\left(\Omega_{0}^{\infty} E ; \mathbb{Q}\right)$ and are dual Hopf algebras. Moreover, the image of $\sigma^{*}$ is contained in the module of primitive elements

$$
\sigma^{*}: H_{\text {free }}^{*}(E) \longrightarrow P\left(H_{\text {free }}^{*}\left(\Omega_{0}^{\infty} E\right)\right)
$$

and dually $\sigma_{*}$ factors over the indecomposable elements of $H_{*}^{\text {free }}\left(\Omega_{0}^{\infty} E\right)$.

Given a pointed space $X$ we have the associated suspension spectrum $S^{\infty} X$ whose $n$-th term is $S^{n} X$ with infinite loop space $\Omega^{\infty} S^{\infty} X$. There is an obvious inclusion $i: X \rightarrow \Omega^{\infty} S^{\infty} X$ inducing a splitting of $\sigma^{*}$ (and $\sigma_{*}$ ):

$$
H^{*}\left(S^{\infty} X\right) \stackrel{\sigma^{*}}{\longrightarrow} \tilde{H}^{*}\left(\Omega_{0}^{\infty} S^{\infty} X\right) \stackrel{i^{*}}{\longrightarrow} \tilde{H}^{*}(X)
$$

is the suspension isomorphism. $\Omega^{\infty} S^{\infty} X$ is the free infinite loop space on $X$ and satisfies the universal property that any pointed map from $X$ to some infinite loop space $Y$ can be extended in a unique way up to homotopy to a map of infinite loop spaces from $\Omega^{\infty} S^{\infty} X$ to $Y$.

The spectra of most relevance to us are $\mathbb{C} P_{-1}^{\infty}$ and the suspension spectrum $S^{\infty} \mathbb{C} P_{+}^{\infty}$ of $\mathbb{C} P^{\infty} \sqcup\{+\}$. We recall the definition of the former. There are two complex vector bundles over the complex projective $n$-space $\mathbb{C} P^{n}$, namely the tautological line bundle $L_{n}$ and its $n$-dimensional complement $L_{n}^{\perp}$ in $\mathbb{C} P^{n} \times \mathbb{C}^{n+1}$. Its Thom space (or one point compactification) is denoted by $\operatorname{Th}\left(L_{n}^{\perp}\right)$. Since the restriction of $L_{n}^{\perp}$ to $\mathbb{C} P^{n-1} \subset \mathbb{C} P^{n}$ is equal to $L_{n-1}^{\perp} \oplus \mathbb{C}$, where $\mathbb{C}$ denotes the trivial line bundle over $\mathbb{C} P^{n-1}$, we get a map

$$
\epsilon: S^{2} \operatorname{Th}\left(L_{n-1}^{\perp}\right) \longrightarrow \operatorname{Th}\left(L_{n}^{\perp}\right) .
$$

The spectrum $\mathbb{C} P_{-1}^{\infty}$ has

$$
\left(\mathbb{C} P_{-1}^{\infty}\right)_{2 n}=\operatorname{Th}\left(L_{n-1}^{\perp}\right), \quad\left(\mathbb{C} P_{-1}^{\infty}\right)_{2 n+1}=S \operatorname{Th}\left(L_{n-1}^{\perp}\right)
$$

and the structure map $\epsilon_{2 n+1}$ is given by the above $\epsilon$. The associated infinite loop space is

$$
\Omega^{\infty} \mathbb{C} P_{-1}^{\infty}=\operatorname{colim}_{n \rightarrow \infty} \Omega^{2 n} \operatorname{Th}\left(L_{n-1}^{\perp}\right) .
$$

The inclusion of $L_{n-1}^{\perp}$ into $L_{n-1}^{\perp} \oplus L_{n-1}=\mathbb{C} P^{n-1} \times \mathbb{C}^{n}$ via the zero section of $L_{n-1}$ induces a map from $\operatorname{Th}\left(L_{n-1}^{\perp}\right)$ into $S^{2 n}\left(\mathbb{C} P_{+}^{n-1}\right)$ and hence a map

$$
\omega: \Omega^{\infty} \mathbb{C} P_{-1}^{\infty} \longrightarrow \Omega^{\infty} S^{\infty}\left(\mathbb{C} P_{+}^{\infty}\right) .
$$

This map fits into a fibration sequence

$$
\Omega^{\infty} \mathbb{C} P_{-1}^{\infty} \stackrel{\omega}{\longrightarrow} \Omega^{\infty} S^{\infty}\left(\mathbb{C} P_{+}^{\infty}\right) \stackrel{\partial}{\longrightarrow} \Omega^{\infty} S^{\infty-1}
$$

where the right-hand term is the direct limit of $\Omega^{n} S^{n-1}[\underline{\mathrm{R}}$. Indeed the inclusion of a fibre $\mathbb{C}^{n} \rightarrow L_{n}^{\perp}$ induces a map $S^{2 n} \rightarrow \operatorname{Th}\left(L_{n}^{\perp}\right)$ and gives rise to a cofibre sequence of spectra $S^{\infty}\left(S^{-2}\right) \rightarrow \mathbb{C} P_{-1}^{\infty} \rightarrow S^{\infty} \mathbb{C} P_{+}^{\infty} \rightarrow S^{\infty}\left(S^{-1}\right)$. (2.3) is the associated fibration sequence of infinite loop spaces.

The component groups of (2.3) are

$$
0 \longrightarrow \mathbb{Z}^{\pi_{0}(\omega)} \longrightarrow \mathbb{Z} \stackrel{\pi_{0}(\partial)}{\longrightarrow} \mathbb{Z} / 2 \longrightarrow 0,
$$

so $\pi_{0}(\omega)$ is multiplication by \pm 2 , depending on the choice of generators. There is a canonical splitting of infinite loop spaces

$$
\Omega^{\infty} S^{\infty}\left(\mathbb{C} P_{+}^{\infty}\right) \simeq \Omega^{\infty} S^{\infty}\left(\mathbb{C} P^{\infty}\right) \times \Omega^{\infty} S^{\infty} .
$$


We fix the generator of $\pi_{0} \Omega^{\infty} S^{\infty}\left(\mathbb{C} P_{+}^{\infty}\right)$ to be the element that maps to +1 under the isomorphisms

$$
\pi_{0}\left(\Omega^{\infty} S^{\infty}\left(\mathbb{C} P_{+}^{\infty}\right)\right) \stackrel{\pi_{0}(c)}{\longrightarrow} \pi_{0}\left(\Omega^{\infty} S^{\infty}\right) \stackrel{\text { degree }}{\longrightarrow} \mathbb{Z},
$$

where $c$ collapses $\mathbb{C} P^{\infty}$ to the non-basepoint of $S^{0}$. We fix the generator of $\pi_{0}\left(\Omega^{\infty} \mathbb{C} P_{-1}^{\infty}\right)$ so that $\pi_{0}(\omega)$ is multiplication by -2 .

2.2. Review of results used. Our divisibility result of Theorem 1.1 is based upon the following three theorems.

Theorem 2.1 ( $[\mathrm{T}])$. The spaces $\mathbb{Z} \times B \Gamma_{\infty}^{+}$and $B \Gamma_{\infty}^{+}\left(=\{0\} \times B \Gamma_{\infty}^{+}\right)$are infinite loop spaces.

Here the superscript $(+)$ denotes Quillen's plus construction; cf. [B]. The product structure can be described as follows. We may view $\Gamma_{g, 2}$ as the mapping class group of surfaces with one incoming and one outgoing boundary component. Gluing the incoming boundary component of one surface to the outgoing component of the other defines a map

$$
\Gamma_{g, 2} \times \Gamma_{h, 2} \longrightarrow \Gamma_{g+h, 2}
$$

and a corresponding map of classifying spaces that makes the disjoint union $\bigsqcup B \Gamma_{g, 2}$ over all $g \geq 0$ into a topological monoid. Consider the map

$$
\bigsqcup_{g \geq 0} B \Gamma_{g, 2} \longrightarrow \mathbb{Z} \times B \Gamma_{\infty}^{+}
$$

that sends $B \Gamma_{g, 2}$ into the component $\{g\} \times B \Gamma_{\infty}^{+}$by the stabilization map (1.1) followed by the map into the plus construction. The infinite loop space structure on $\mathbb{Z} \times B \Gamma_{\infty}^{+}$is compatible with the monoidal structure on $\bigsqcup B \Gamma_{g, 2}$, and the induced map

$$
\Omega B\left(\bigsqcup_{g \geq 0} B \Gamma_{g, 2}\right) \longrightarrow \mathbb{Z} \times B \Gamma_{\infty}^{+}
$$

is a homotopy equivalence of loop spaces. We refer to $T$ for details.

To state the next result, for each prime $p$ we pick a positive integer $k=k(p)$ so that $-k$ reduces to a generator of the units $\left(\mathbb{Z} / p^{2}\right)^{\times}$when $p$ is odd. We pick $k=5$ when $p=2$. Write $\psi^{-k}$ for the self-map of $\mathbb{C} P^{\infty}$ that multiplies by $-k$ on the second cohomology group. Composing with the inclusion into $\Omega^{\infty} S^{\infty}\left(\mathbb{C} P^{\infty}\right)$ and using the loop sum we have a map

$$
1+k \psi^{-k}: \mathbb{C} P^{\infty} \longrightarrow \Omega^{\infty} S^{\infty}\left(\mathbb{C} P^{\infty}\right)
$$

and, using the universal property of free infinite loop spaces, a unique extension to a self-map of $\Omega^{\infty} S^{\infty}\left(\mathbb{C} P^{\infty}\right)$, again denoted $1+k \psi^{-k}$.

Theorem 2.2. There are infinite loop maps

$$
\alpha: \mathbb{Z} \times B \Gamma_{\infty}^{+} \longrightarrow \Omega^{\infty} \mathbb{C} P_{-1}^{\infty}, \quad \mu_{p}: \Omega^{\infty} S^{\infty}\left(\mathbb{C} P_{+}^{\infty}\right) \longrightarrow\left(\mathbb{Z} \times B \Gamma_{\infty}^{+}\right)_{p}^{\wedge}
$$

such that the composition $\omega \circ \alpha \circ \mu_{p}$ and the self-map

$$
\left(\begin{array}{cc}
1+k \psi^{-k} & 0 \\
0 & -2
\end{array}\right): \Omega^{\infty} S^{\infty} \mathbb{C} P^{\infty} \times \Omega^{\infty} S^{\infty} \longrightarrow \Omega^{\infty} S^{\infty} \mathbb{C} P^{\infty} \times \Omega^{\infty} S^{\infty}
$$

become homotopic after -adic completion. 
This is an improvement on the main theorem of [MT], where the map in the lower left corner had been left undetermined. For our calculations in Section 4.3 we need this map however to be zero. A proof of Theorem 2.2 is given in the final Section 5 .

Remark 2.3. The reader is referred to $\mathrm{BK}$ for the notion of $p$-adic completion (also called $\mathbb{F}_{p}$-completion). For connected, compact CW-complexes $X$ and infinite loop spaces $\Omega^{\infty} E$ of finite type one has

$$
\left[X,\left(\Omega^{\infty} E\right)_{p}^{\wedge}\right]=\left[X, \Omega^{\infty} E\right] \otimes \mathbb{Z}_{p}, \quad H^{*}\left(\left(\Omega^{\infty} E\right)_{p}^{\wedge} ; \mathbb{Z}\right)=H^{*}\left(\Omega^{\infty} E ; \mathbb{Z}_{p}\right) .
$$

Furthermore, note that the homotopy class of the map $\alpha$ in Theorem 2.2 is uniquely determined by its composition with $\mathbb{Z} \times B \Gamma_{\infty} \rightarrow \mathbb{Z} \times B \Gamma_{\infty}^{+}$. Indeed since $\Omega^{\infty} \mathbb{C} P_{-1}^{\infty}$ is an infinite loop space the induced map

$$
\left[\mathbb{Z} \times B \Gamma_{\infty}^{+}, \Omega^{\infty} \mathbb{C} P_{-1}^{\infty}\right] \longrightarrow\left[\mathbb{Z} \times B \Gamma_{\infty}, \Omega^{\infty} \mathbb{C} P_{-1}^{\infty}\right]
$$

is an isomorphism. This is a standard property of the plus construction; cf. [B].

Before we give a detailed description of $\alpha$ in the next section, we state here the third result.

Theorem 2.4 ([MW]). The map $\alpha$ is a homotopy equivalence.

\section{Characteristic Classes of Surface Bundles}

3.1. Universal surface bundles. The methods used in this and the surrounding papers do not use the mapping class groups directly but rather the topological groups of orientation-preserving diffeomorphisms of surfaces. We briefly review the correspondence.

Let $F_{g, b}$ be a connected surface of genus $g$ with $b$ boundary circles. We write $\operatorname{Diff}\left(F_{g, b} ; \partial\right)$ for the topological group of orientation-preserving diffeomorphisms that keep (a neighborhood of) the boundary pointwise fixed. For $g \geq 2$, results from [EE] and [ES] yield

$$
B \Gamma_{g, b} \simeq B \operatorname{Diff}\left(F_{g, b} ; \partial\right)
$$

so that $B \Gamma_{g, b}$ classifies diffeomorphism classes of smooth fibre bundles $\pi: E \rightarrow X$ with fibre $F_{g, b}$ and standard boundary behavior:

$$
\partial E=X \times \bigsqcup_{1}^{b} S^{1}, \quad \pi \mid \partial E=\operatorname{proj}_{X} .
$$

Similarly,

$$
B \Gamma_{g, b}^{s} \simeq B \operatorname{Diff}\left(F_{g, b} ; \partial \sqcup\left\{x_{1}, \ldots, x_{s}\right\}\right)
$$

where $x_{1}, \ldots, x_{s}$ are distinct interior points of $F_{g, b}$. Take $s=1$. Since $\operatorname{Diff}\left(F_{g, b} ; \partial\right)$ acts transitively on the interior of $F_{g, b}$,

$$
E\left(F_{g, b}\right):=E \operatorname{Diff}\left(F_{g, b} ; \partial\right) \times_{\operatorname{Diff}\left(F_{g, b} ; \partial\right)} F_{g, b} \simeq B \operatorname{Diff}\left(F_{g, b} ; \partial \sqcup\{x\}\right) \simeq B \Gamma_{g, b}^{1} .
$$

The forgetful map $\pi: B \Gamma_{g, b}^{1} \rightarrow B \Gamma_{g, b}$ corresponds to the universal smooth $F_{g, b}$ bundle

$$
F_{g, b} \longrightarrow E\left(F_{g, b}\right) \longrightarrow B \operatorname{Diff}\left(F_{g, b} ; \partial\right) .
$$

The central extension (1.2) is classified by "the differential at $x$ ",

$$
\operatorname{Diff}\left(F_{g, b} ; \partial \sqcup\{x\}\right) \longrightarrow \mathrm{GL}^{+}\left(T_{x} F_{g, b}\right) \simeq S O(2) .
$$


Hence the circle bundle induced from (1.2) by applying the classifying space functor corresponds to the circle bundle of the vertical tangent bundle associated with (3.1).

3.2. The map $\alpha$ and the kappa classes. The map of infinite loop spaces $\alpha: \mathbb{Z} \times B \Gamma_{\infty}^{+} \rightarrow \Omega^{\infty} \mathbb{C} P_{-1}^{\infty}$ constructed in section 2 of [MT] restricts to a map $\alpha_{g, 2}: B \Gamma_{g, 2} \rightarrow \Omega_{g}^{\infty} \mathbb{C} P_{-1}^{\infty}$ that is homotopic to the composition

$$
\alpha_{g, 2}: B \Gamma_{g, 2} \longrightarrow B \Gamma_{g+1} \stackrel{\alpha_{g+1}}{\longrightarrow} \Omega_{g}^{\infty} \mathbb{C} P_{-1}^{\infty},
$$

where the left-hand map is induced from gluing the two parametrized boundary circles together. These maps are up to homotopy compatible with the monoidal structure on $\bigsqcup B \Gamma_{g, 2}$.

We next recall a description of $\alpha_{g+1}$ which is well-suited for identifying the kappa classes. Let $\pi: E \rightarrow X$ be a smooth surface bundle with closed fiber $F$. Thus $E=P \times \times_{\operatorname{Diff}(F)} F$ where $P$ is a principal $\operatorname{Diff}(F)$ bundle over $X$. We do not assume that $X$ is smooth or finite dimensional, only that $X$ is paracompact (or a CW-complex).

We denote by $\operatorname{Emb}\left(F, \mathbb{R}^{n}\right)$ the space of smooth embeddings in the $C^{\infty}$-topology, and let $\mathbb{R}^{\infty}$ and $\operatorname{Emb}\left(F, \mathbb{R}^{\infty}\right)$ be the colimits of $\mathbb{R}^{n}$ and $\operatorname{Emb}\left(F, \mathbb{R}^{n}\right)$, respectively. We shall consider fiberwise embeddings $\iota: E \rightarrow X \times \mathbb{R}^{\infty}$, that is, fiberwise maps such that each $\iota_{x}: E_{x} \rightarrow\{x\} \times \mathbb{R}^{\infty}$ is an embedding and such that the adjoint $\operatorname{Diff}(F)$-equivariant map $P \rightarrow \operatorname{Emb}\left(F, \mathbb{R}^{\infty}\right)$ is continuous. Such an $\iota$ is equivalent to a section of $P \times_{\operatorname{Diff}(F)} \operatorname{Emb}\left(F, \mathbb{R}^{\infty}\right)$. Note that $\operatorname{Emb}\left(F, \mathbb{R}^{\infty}\right)$ is contractible so that such a section always exists.

An embedding $\iota_{x}: F \hookrightarrow \mathbb{R}^{n+2}$ extends to a map from the normal bundle $N^{n} \iota_{x}=\left\{(p, v) \mid v \perp T_{p} F\right\}$ into $\mathbb{R}^{n+2}$ by sending $(p, v)$ to $p+v$. (Here we have identified $F$ with its image under $\iota_{x}$.) We call the embedding $\iota_{x}$ fat if this map restricts to an embedding of the unit disk bundle $D\left(N^{n} \iota_{x}\right)$. The subspace of fat embeddings $\operatorname{Emb}^{f}\left(F, \mathbb{R}^{\infty}\right) \subset \operatorname{Emb}\left(F, \mathbb{R}^{\infty}\right)$ is contractible, since the inclusion is a homotopy equivalence by the tubular neighborhood theorem and since $\operatorname{Emb}\left(F, \mathbb{R}^{\infty}\right)$ is contractible by Whitney's embedding theorem. A fibrewise fat embedding $\iota: E \rightarrow$ $X \times \mathbb{R}^{\infty}$ is then a section of the fibre bundle $P \times_{\operatorname{Diff}(F)} \operatorname{Emb}^{f}\left(F, \mathbb{R}^{\infty}\right)$.

Suppose first that $\iota: E \rightarrow X \times \mathbb{R}^{n+2}$ is a fibrewise fat embedding of codimension $n$. The Pontryagin-Thom construction associates a "collapse" map onto the Thom space of the fibrewise normal bundle,

$$
c_{\pi, \iota}: X_{+} \wedge S^{n+2} \longrightarrow D\left(N_{\pi}^{n} \iota\right) / S\left(N_{\pi}^{n} \iota\right)=\operatorname{Th}\left(N_{\pi}^{n} \iota\right) .
$$

We are particularly interested in its adjoint map $X \rightarrow \Omega^{n+2} \operatorname{Th}\left(N_{\pi}^{n} \iota\right)$.

Let $G(2, n)$ be the Grassmann manifold of oriented 2-dimensional subspaces of $\mathbb{R}^{n+2}$, and let $U_{n}$ and $U_{n}^{\perp}$ be the two complementary universal bundles over it of dimension 2 and $n$, respectively. The fat embedding $\iota$ induces bundle maps

$$
T_{\pi} E \longrightarrow U_{n}, \quad N_{\pi}^{n} \iota \longrightarrow U_{n}^{\perp}
$$

and a commutative diagram

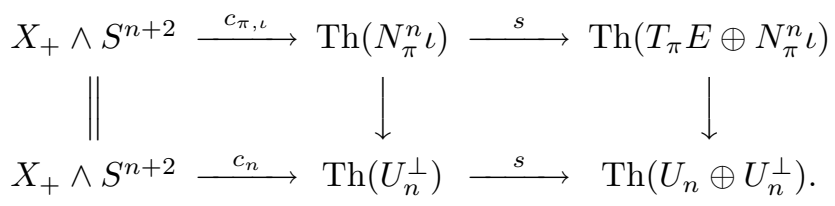


In the general case of a fiberwise fat embedding $\iota: E \rightarrow X \times \mathbb{R}^{\infty}$, the base space $X$ is the colimit of the subspaces

$$
X_{n}:=\left\{x \in X \mid \iota_{x}\left(E_{x}\right) \subset\{x\} \times \mathbb{R}^{n+2}\right\},
$$

and the diagram

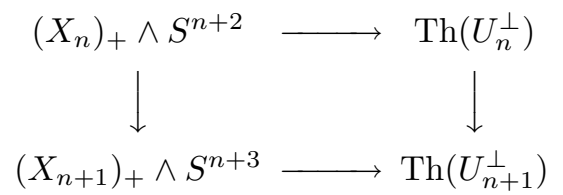

is commutative since $\left.U_{n+1}^{\perp}\right|_{G(2, n)}=U_{n}^{\perp}$. Taking adjoints we get

$$
\alpha_{\pi, \iota}: X \longrightarrow \operatorname{colim}_{n \rightarrow \infty} \Omega^{n+2} \operatorname{Th}\left(U_{n}^{\perp}\right) .
$$

Since $\operatorname{Emb}^{f}\left(F, \mathbb{R}^{\infty}\right)$ is contractible, all sections of $P \times_{\operatorname{Diff}(F)} \operatorname{Emb}^{f}\left(F, \mathbb{R}^{\infty}\right)$ are homotopic, and consequently the homotopy class $\left[\alpha_{\pi, \iota}\right]$ is independent of the choice of $\iota$. We will therefore from now on suppress the subscript $\iota$.

Realification gives a $(2 n-1)$-connected map from $\mathbb{C} P^{n}$ into the oriented Grassmannian $G(2,2 n)$ covered by a bundle map $L_{n}^{\perp} \rightarrow U_{2 n}^{\perp}$. Thus $G(2, \infty) \simeq \mathbb{C} P^{\infty}$ and

$$
\Omega^{\infty} \mathbb{C} P_{-1}^{\infty}=\operatorname{colim} \Omega^{2 n+2} \operatorname{Th}\left(L_{n}^{\perp}\right) \stackrel{\simeq}{\longrightarrow} \operatorname{colim} \Omega^{2 n+2} \operatorname{Th}\left(U_{2 n}^{\perp}\right)
$$

is a homotopy equivalence. Altogether we have a well-defined homotopy class

$$
\alpha_{\pi}: X \longrightarrow \Omega^{\infty} \mathbb{C} P_{-1}^{\infty}
$$

For $X=B \operatorname{Diff}\left(F_{g+1}\right) \simeq B \Gamma_{g+1}$ this is the map $\alpha_{g+1}$ of (3.2).

Let us check that the image of $\alpha_{g+1}$, and hence the image of $\alpha_{g, 2}$, lie in the $g$-component of $\Omega^{\infty} \mathbb{C} P_{-1}^{\infty}$, or equivalently that the composition

$$
\text { proj } \circ \omega \circ \alpha_{g+1}: B \Gamma_{g+1} \longrightarrow \Omega^{\infty} S^{\infty}\left(\mathbb{C} P_{+}^{\infty}\right) \longrightarrow \Omega^{\infty} S^{\infty}
$$

lands in the $-2 g$ component (with identification of components chosen at the end of Section 2.1). Consider (3.3) with $X$ a single point and $E=F_{g+1}$. The bottom row in (3.3) is thus $s \circ c_{n}: S^{n+2} \rightarrow G(2,2 n)_{+} \wedge S^{n+2}$, and we need to compute the degree of the composition of this map with the projection onto $S^{n+2}$. This degree is given by the evaluation of the pullback of the generator of $H^{n+2}\left(S^{n+2}\right)$ on the fundamental class $\left[S^{n+2}\right]$. Under the projection the fundamental class is pulled back to the Thom class of the trivial bundle $U_{n} \oplus U_{n}^{\perp}$. Writing $\lambda_{U}$ for the Thom class of the vector bundle $U$, the degree is thus given by

$$
\begin{aligned}
\left\langle c_{\pi}^{*} s^{*}\left(\lambda_{U_{n}} \cdot \lambda_{U_{n}^{\perp}}\right),\left[S^{2 n+2}\right]\right\rangle & =\left\langle c_{\pi}^{*}\left(e\left(T F_{g+1}\right) \cdot \lambda_{U_{n}^{\perp}}\right),\left[S^{2 n+2}\right]\right\rangle \\
& =\left\langle e\left(T F_{g+1}\right),\left[F_{g+1}\right]\right\rangle=-2 g,
\end{aligned}
$$

as claimed.

Next we compute the maps $\alpha_{g, 2}$ and $\alpha_{g+1,2}$ under the map $B \Gamma_{g, 2} \rightarrow B \Gamma_{g+1,2}$ induced from gluing a torus with two boundary circles $F_{1,2}$ to $F_{g, 2}$. Considering $F_{1,2}$ as a fibre bundle over a point the construction above gives an element [1] $\epsilon$ $\Omega_{1}^{\infty} \mathbb{C} P_{-1}^{\infty}$. Loop sum with [1] in $\Omega^{\infty} \mathbb{C} P_{-1}^{\infty}$ translates the $g$-component into the 
$(g+1)$-component and

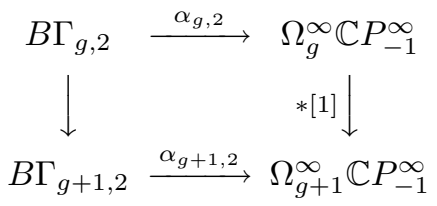

is homotopy commutative. To see this observe that the left vertical map is multiplication by the basepoint of $B \Gamma_{1,2}$ in the monoid $\bigsqcup_{g \geq 0} B \Gamma_{g, 2}$ and the right vertical map is multiplication by its image in $\Omega_{1}^{\infty} \mathbb{C} P_{-1}^{\infty}$. Homotopy commutativity of diagram (3.4) now follows because the $\alpha_{g, 2}$ induce a map of monoids up to homotopy. This is because $\alpha$ is a map of infinite loop spaces, $\alpha$ restricts to $\alpha_{g, 2}$ on $B \Gamma_{g, 2}$, and the infinite loop space structure on $\mathbb{Z} \times B \Gamma_{\infty}^{+}$is compatible with the monoidal structure. (Alternatively, homotopy commutativity of (3.4) follows from a calculation of pretransfers; cf. G2.)

Let $\tilde{\alpha}$ denote the restriction of $\alpha$ in Theorem 2.2 to the zero component. Restricted to $B \Gamma_{g, 2}$ it is homotopic to $\tilde{\alpha}_{g, 2}=(*[-g]) \circ \alpha_{g, 2}$. We can now relate the kappa classes to spectrum cohomology. Consider

$$
B \Gamma_{g, 2} \stackrel{\tilde{\alpha}_{g, 2}}{\longrightarrow} \Omega_{0}^{\infty} \mathbb{C} P_{-1}^{\infty} \stackrel{\omega}{\longrightarrow} \Omega_{0}^{\infty} S^{\infty}\left(\mathbb{C} P_{+}^{\infty}\right)
$$

and recall the cohomology suspension from Section 2.1:

$$
\sigma^{*}: H^{2 i}\left(\mathbb{C} P^{\infty} ; \mathbb{Z}\right) \simeq H^{2 i}\left(S^{\infty} \mathbb{C} P_{+}^{\infty}\right) \longrightarrow H^{2 i}\left(\Omega_{0}^{\infty} S^{\infty}\left(\mathbb{C} P_{+}^{\infty}\right)\right) .
$$

Theorem 3.1. The Miller-Morita-Mumford class $\kappa_{i}$ is equal to $(\omega \circ \tilde{\alpha})^{*}\left(\sigma^{*} e^{i}\right)$, where $e \in H^{2}\left(\mathbb{C} P^{\infty} ; \mathbb{Z}\right)$ is the Euler class of the canonical line bundle.

Proof. Let $\pi: E \rightarrow X$ be a smooth fibre bundle with fibre $F_{g+1}$, classified by $f_{\pi}: X \rightarrow B \Gamma_{g+1}$. By definition

$$
f_{\pi}^{*}\left(\kappa_{i}\right)=\pi_{!}\left(e\left(T_{\pi} E\right)^{i+1}\right) \in H^{2 i}(X ; \mathbb{Z}),
$$

where $\pi_{!}$is the composition of the Thom isomorphism and the Pontrjagin-Thom collapse map

$$
\tilde{H}^{2 i+2}(E ; \mathbb{Z}) \stackrel{\simeq}{\longrightarrow} \tilde{H}^{2 i+2 n+2}\left(\operatorname{Th}\left(N_{\pi}^{n} \iota\right) ; \mathbb{Z}\right) \stackrel{c_{\pi}^{*}}{\longrightarrow} H^{2 i+2 n+2}\left(S^{2 n+2} \wedge X_{+} ; \mathbb{Z}\right)
$$

followed by the $(2 n+2)$-nd desuspension; here the notation is as in (3.3). Let $x \in H^{k}\left(\mathbb{C} P^{N} ; \mathbb{Z}\right) \simeq H^{k}(G(2,2 N) ; \mathbb{Z})$ for $N \gg k$. The $(2 n+2)$-fold suspension $\Sigma^{2 n+2}(x) \in H^{k+2 n+2}\left(S^{2 n+2} \wedge \mathbb{C} P^{N}, \mathbb{Z}\right)$ is $x$ times the Thom class of the trivial $2 n+2$ real bundle $L_{n}^{\perp} \oplus L_{n}$. Thus

$$
s^{*}\left(\Sigma^{2 n+2}(x)\right)=s^{*}\left(\lambda_{L_{n}^{\perp}} \cdot \lambda_{L_{n}} \cdot x\right)=\lambda_{L_{\frac{1}{n}}} \cdot e\left(L_{n}\right) \cdot x .
$$

To interpret these formulas, recall that the cohomology of the Thom space of a vector bundle is a module over the cohomology of the base space, and a map of bundles (such as $s$ ) induces a map of modules. Furthermore, as elements in the cohomology of the Thom space of the trivial bundle, $\lambda_{L_{n}^{\perp}}$ and $\lambda_{L_{n}}$ are pulled back along the bundle projections $\pi_{1}$ and $\pi_{2}$ of $L_{n}^{\perp} \oplus L_{n}$ onto the first and second summands. Hence, as $\pi_{1} \circ s$ is the identity, we have $s^{*}\left(\lambda_{L_{n}^{\perp}}\right)=\lambda_{L_{n}^{\perp}}$, and as $\pi_{2} \circ s$ factors through the base space, $s^{*}\left(\lambda_{L_{n}}\right)=1 \cdot e\left(L_{n}\right)$. Finally, take $x=e^{i}$ in the above formula and use the commutativity of (3.3) to complete the proof. 
3.3. One part of Akita's conjecture. For our next theorem we need the relation between Steenrod operations and characteristic classes of vector bundles. Recall the $i$-th Steenrod operation:

$$
\begin{aligned}
& P^{i}: H^{k}\left(X ; \mathbb{F}_{p}\right) \longrightarrow H^{k+2 i(p-1)}\left(X ; \mathbb{F}_{p}\right), \quad p \text { odd }, \\
& S q^{i}: H^{k}\left(X, \mathbb{F}_{2}\right) \longrightarrow H^{k+i}\left(X, \mathbb{F}_{2}\right) .
\end{aligned}
$$

Let $E$ be an oriented vector bundle over $X$ and $\lambda_{U}$ its cohomology Thom class. One defines $v_{i}(E) \in H^{2 i(p-1)}\left(X ; \mathbb{F}_{p}\right)$, respectively $v_{i}(E) \in H^{i}\left(X ; \mathbb{F}_{2}\right)$, by

$$
P^{i}\left(\lambda_{E}\right)=v_{i}(E) \lambda_{E}, \quad S q^{i}\left(\lambda_{E}\right)=v_{i}(E) \lambda_{E} .
$$

For $p=2$, these are the Stiefel-Whitney classes, and for $p$ odd they were first defined by Wu; cf. MSt. For an oriented 2-plane bundle (or complex line bundle) $L$,

$$
\begin{array}{cc}
v_{1}(L)=e(L)^{p-1} & \text { for } p \text { odd } \\
v_{2}(L)=e(L) \quad \text { and } \quad v_{1}(L)=0 & \text { for } p=2 .
\end{array}
$$

Moreover, the total class

$$
v(E)=1+v_{1}(E)+v_{2}(E)+\cdots \in H^{*}\left(X ; \mathbb{F}_{p}\right)
$$

takes direct sums of oriented vector bundles into (graded) products.

Theorem 3.2. The modulo $p$ reduction of $\kappa_{i} \in H^{2 i}\left(B \Gamma_{\infty} ; \mathbb{F}_{p}\right)$ is zero when $i+1 \equiv$ $0(\bmod p-1)$.

Proof 3 Let $H(\mathbb{Z}, k)$ denote the Eilenberg-MacLane space with non-trivial homotopy $\mathbb{Z}$ in dimension $k$. The Thom class $\lambda_{n}=\lambda_{L_{n}^{\perp}}$ is represented by a map from $\operatorname{Th}\left(L_{n}^{\perp}\right)$ to $H(\mathbb{Z}, 2 n)$. We let $Y_{2 n+2}$ be its homotopy fibre, so that there is a fibration sequence

$$
Y_{2 n+2} \stackrel{j_{2 n+2}}{\longrightarrow} \operatorname{Th}\left(L_{n}^{\perp}\right) \stackrel{\lambda_{n}}{\longrightarrow} H(\mathbb{Z}, 2 n) .
$$

The spaces $Y_{2 n+2}$ are the $(2 n+2)$-nd terms of a spectrum $Y$ and the $j_{2 n+2}$ define a map $j: Y \rightarrow \mathbb{C} P_{-1}^{\infty}$ of spectra. Since $\Omega^{2 n+2} H(\mathbb{Z}, 2 n)$ has vanishing homotopy groups,

$$
\Omega^{\infty} Y \stackrel{\Omega^{\infty} j}{\longrightarrow} \Omega^{\infty} \mathbb{C} P_{-1}^{\infty}
$$

is a (weak) homotopy equivalence. Thus the bottom vertical map in the commutative diagram

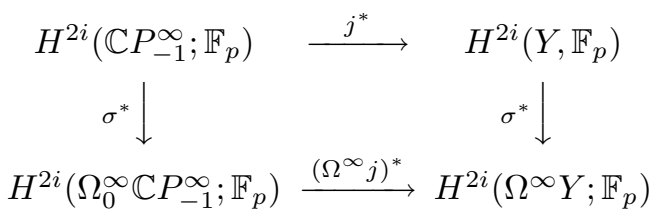

is an isomorphism. The proof of Theorem 3.1 shows that

$$
\tilde{\alpha}^{*} \sigma^{*}\left(e^{i+1} \lambda_{L^{\perp}}\right)=\kappa_{i},
$$

so it suffices to prove that $j^{*}$ vanishes when $i+1 \equiv 0(\bmod p-1)$. Equivalently, we must show that $e^{i+1} \lambda_{n}$ is in the image of

$$
\lambda_{n}^{*}: H^{*}\left(H(\mathbb{Z}, 2 n) ; \mathbb{F}_{p}\right) \longrightarrow H^{*}\left(\operatorname{Th}\left(L_{n}^{\perp}\right) ; \mathbb{F}_{p}\right)
$$

\footnotetext{
${ }^{3}$ We thank John Rognes for this proof; it replaces a more cumbersome earlier argument.
} 
in the stated dimensions. This is implied by

$$
v\left(L_{n}^{\perp}\right)=v\left(L_{n}\right)^{-1}=\left\{\begin{aligned}
\left(1+e^{p-1}\right)^{-1}, & p>2, \\
(1+e)^{-1}, & p=2 .
\end{aligned}\right.
$$

Since $P^{i}\left(\lambda_{n}\right) \in$ image $\left(\lambda_{n}^{*}\right)$ the result follows.

Remark 3.3. The relation $P^{i}\left(\lambda_{L^{\perp}}\right)=\kappa_{i(p-1)-1} \lambda_{L^{\perp}}$ used above is further exploited in G2 to define secondary classes $\mu_{i}$ with $p \mu_{i}=\kappa_{i(p-1)-1}$ in cohomology with $\mathbb{Z} / p^{2} \mathbb{Z}$ coefficients.

Theorem 3.2 proves half of Akita's, Theorem 1.2. The other half is implied by Theorem 1.1, which is proved below.

\section{Proof of Theorem 1.1}

4.1. Segal's splitting. The inclusion of $\mathbb{C} P^{\infty}$ in $B U$ that represents the reduced canonical line bundle (of virtual dimension zero) extends to a map of infinite loop spaces

$$
l: \Omega^{\infty} S^{\infty}\left(\mathbb{C} P^{\infty}\right) \longrightarrow B U
$$

by Bott periodicity and the universal property of the free infinite loop space functor $\Omega^{\infty} S^{\infty}$. Graeme Segal [S] proved that this map has a splitting. In [C] Michael Crabb gives a construction of this map as the $S^{1}$-equivariant $J$-homomorphism.

Theorem 4.1 ([S], $[\mathrm{C}])$. The map $l$ has a left inverse up to homotopy. In the resulting decomposition

$$
\Omega^{\infty} S^{\infty}\left(\mathbb{C} P^{\infty}\right) \simeq B U \times F i b(l)
$$

the homotopy fiber Fib(l) has vanishing rational cohomology.

In particular, this gives an identification of Hopf algebras

$$
H_{\text {free }}^{*}\left(\Omega^{\infty} S^{\infty}\left(\mathbb{C} P^{\infty}\right)\right) \simeq H^{*}(B U ; \mathbb{Z})
$$

induced by $l^{*}$. The graded module of primitive elements of the right-hand side is a copy of $\mathbb{Z}$ in each even degree generated by the integral Chern character class $s_{i}=i ! c h_{i}$. Since $l \circ i$ represents the (reduced) line bundle and $c h_{i}(L)=\frac{1}{i !} e^{i},(4.1)$ implies that

$$
i^{*}: H_{\text {free }}^{*}\left(\Omega^{\infty} S^{\infty}\left(\mathbb{C} P^{\infty}\right)\right) \longrightarrow H^{*}\left(\mathbb{C} P^{\infty} ; \mathbb{Z}\right)
$$

sends the primitive generator $s_{i}$ to $e^{i}$. Note from (2.2) that we also have that

$$
H^{*}\left(\mathbb{C} P^{\infty} ; \mathbb{Z}\right)=H^{*}\left(S^{\infty} \mathbb{C} P^{\infty} ; \mathbb{Z}\right) \stackrel{\sigma^{*}}{\longrightarrow} P\left(H_{\text {free }}^{*}\left(\Omega^{\infty} S^{\infty}\left(\mathbb{C} P^{\infty}\right)\right)\right)
$$

maps $e^{i}$ to $s_{i}$. Therefore, Theorem 3.1 translates into

$$
\kappa_{i}=(l \circ \omega \circ \alpha)^{*}\left(s_{i}\right) .
$$

The map $\omega$ in (2.3) induces an isomorphism on rational cohomology because the cohomology of $\Omega^{\infty} S^{\infty-1}$ is well known to be all torsion. The isomorphisms

$$
H^{*}(B U ; \mathbb{Q}) \stackrel{l^{*}}{\longrightarrow} H^{*}\left(\Omega_{0}^{\infty} S^{\infty}\left(\mathbb{C} P_{+}^{\infty}\right) ; \mathbb{Q}\right) \stackrel{\omega^{*}}{\longrightarrow} H^{*}\left(\Omega^{\infty} \mathbb{C} P_{-1}^{\infty} ; \mathbb{Q}\right)
$$

together with Theorem 2.4 thus give an isomorphism

$$
H^{*}\left(B \Gamma_{\infty} ; \mathbb{Q}\right) \simeq H^{*}(B U ; \mathbb{Q})
$$


of Hopf algebras. Hence $P\left(H_{\text {free }}^{*}\left(B \Gamma_{\infty}\right)\right)$ is a copy of $\mathbb{Z}$ in each even degree. We choose a generator

$$
\tau_{i} \in P\left(H_{\text {free }}^{2 i}\left(B \Gamma_{\infty}\right)\right)
$$

such that $\kappa_{i}=D_{i} \tau_{i}$ for some positive number $D_{i}$. The next section gives close upper and lower bounds for $D_{i}$.

4.2. A lower and an upper bound. Recall the definition of the symplectic characteristic classes for surface bundles. The action of $\Gamma_{g, 1}$ on $H^{1}\left(F_{g, 1} ; \mathbb{Z}\right)=\mathbb{Z}^{2 g}=$ $H^{1}\left(F_{g} ; \mathbb{Z}\right)$ induces the standard symplectic representation, and hence a representation of $\Gamma_{g, 2}$ via the map $\Gamma_{g, 2} \rightarrow \Gamma_{g, 1}$ induced by gluing a disk to one of the boundary components. We may let $g \rightarrow \infty$ and obtain

$$
B \Gamma_{\infty} \longrightarrow B \operatorname{Sp}(\mathbb{Z})
$$

This map can be composed with the map into $B \operatorname{Sp}(\mathbb{R}) \simeq B U$ so that we have a map

$$
\eta: B \Gamma_{\infty} \longrightarrow B U
$$

Theorem $4.2\left([\mathrm{Mo},[\mathrm{Mu}])\right.$. In $H^{*}\left(B \Gamma_{\infty} ; \mathbb{Q}\right)$ one has the relation

$$
\eta^{*}\left(s_{2 i-1}\right)=(-1)^{i}\left(\frac{B_{i}}{2 i}\right) \kappa_{2 i-1} .
$$

We are now in a position to prove

Theorem 4.3. For all $i \geq 1, D_{2 i}=2$ and

$$
D_{2 i-1}=\operatorname{den}\left(\frac{B_{i}}{2 i}\right) \quad \text { or } \quad D_{2 i-1}=2 \operatorname{den}\left(\frac{B_{i}}{2 i}\right)
$$

Proof. As $s_{2 i-1}$ and $\kappa_{2 i-1}$ are integral classes, Theorem 4.2 implies immediately that modulo torsion $\operatorname{den}\left(B_{i} / 2 i\right)$ divides $\kappa_{2 i-1}$. By Theorem 3.2 (for $p=2$ ) we also know that 2 divides $\kappa_{2 i}$. This establishes the lower bounds for all $i \geq 1$. The upper bounds are a consequence of Theorem 2.2 as we explain now.

As in Section 2.2, let $k$ be a positive integer such that $-k$ generates $\left(\mathbb{Z} / p^{2}\right)^{\times}$for odd $p$ and let $k=5$ when $p=2$. Then by Theorem 2.2 there is a factorization

$$
1+k \psi^{-k}: \mathbb{C} P^{\infty} \stackrel{\mu_{p}}{\longrightarrow}\left(B \Gamma_{\infty}^{+}\right)_{p}^{\wedge} \stackrel{\omega \circ \alpha}{\longrightarrow} \Omega_{0}^{\infty} S^{\infty}\left(\mathbb{C} P_{+}^{\infty}\right)_{p}^{\wedge} \stackrel{\text { proj }}{\longrightarrow} \Omega^{\infty} S^{\infty}\left(\mathbb{C} P^{\infty}\right)_{p}^{\wedge}
$$

Since $\Omega^{\infty} S^{\infty}\left(\mathbb{C} P^{\infty}\right)$ is of finite type,

$$
H_{\text {free }}^{*}\left(\Omega^{\infty} S^{\infty}\left(\mathbb{C} P^{\infty}\right)_{p}^{\wedge}\right)=H_{\text {free }}^{*}\left(\Omega^{\infty} S^{\infty}\left(\mathbb{C} P^{\infty}\right)\right) \otimes \mathbb{Z}_{p}
$$

It follows from $(4.2)$ that $\mu_{p}^{*}\left(\kappa_{i}\right)$ is the image of the primitive generator $s_{i}$ under

$$
\left(1+k \psi^{-k}\right)^{*}: P H_{\text {free }}^{2 i}\left(\Omega^{\infty} S^{\infty}\left(\mathbb{C} P^{\infty}\right)\right) \otimes \mathbb{Z}_{p} \longrightarrow H^{2 i}\left(\mathbb{C} P^{\infty} ; \mathbb{Z}_{p}\right) .
$$


Both groups are copies of $\mathbb{Z}_{p}$ and $\left(1+k \psi^{-k}\right)^{*}$ in dimension $2 j$ is multiplication by $1+k(-k)^{j}=1-(-k)^{j+1}$. Therefore, $\nu_{p}\left(D_{j}\right) \leq \nu_{p}\left(1-(-k)^{j+1}\right)$.

We have the following well-known table of $p$-adic valuations (see, for example, lemma 2.12 in $\underline{\mathrm{A} 2}$ ):

$$
\begin{aligned}
& \nu_{p}\left(1-(-k)^{s}\right)=\left\{\begin{array}{rll}
1+\nu_{p}(s) & \text { if } s \equiv 0 & \bmod (p-1), p \text { odd } \\
0 & \text { if } s \neq 0 \quad & \bmod (p-1), p \text { odd }
\end{array}\right. \\
& \nu_{2}\left(1-(-k)^{s}\right)=\left\{\begin{array}{rll}
2+\nu_{p}(s) & \text { if } s \equiv 0 & \bmod (2) \\
1 & \text { if } s \not \equiv 0 & \bmod (2)
\end{array}\right.
\end{aligned}
$$

When $j=2 i$, for $p$ odd, $\nu_{p}\left(1-(-k)^{j+1}\right)$ is zero and for $p=2$ it is equal to 1 . This gives $D_{2 i} \leq 2$. When $j=2 i-1$, for $p$ odd we have $\nu_{p}\left(1-(-k)^{j+1}\right)=1+\nu_{p}(2 i)$ if $2 i$ divides $p-1$ and zero otherwise. This is precisely $\nu_{p}\left(\operatorname{den}\left(B_{i} / 2 i\right)\right)$ (compare (1.3)). If $p=2$, however, $\nu_{2}\left(1-(-k)^{j+1}\right)=2+\nu_{2}(2 i)$, which is one more than $\nu_{2}\left(\operatorname{den}\left(B_{i} / 2 i\right)\right)$. This gives $D_{2 i-1} \leq 2 \operatorname{den}\left(B_{i} / 2 i\right)$.

4.3. The final factor of 2. Theorem 4.3 leaves us with an indeterminacy of a factor of 2 in the odd case, and Theorem 1.1 will follow immediately from Theorem 4.5 below. The proof requires several extra results: the improvement of the main theorem from [MT] as stated in Theorem 2.2 (for $p=2$ ), Theorem 2.4, as well as part of theorem 1.3 from G1, which we state as

Theorem 4.4. $H_{*}\left(\Omega_{0}^{\infty} \mathbb{C} P_{-1}^{\infty} ; \mathbb{F}_{2}\right) \stackrel{\omega_{*}}{\longrightarrow} H_{*}\left(\Omega_{0}^{\infty} S^{\infty}\left(\mathbb{C} P_{+}^{\infty}\right) ; \mathbb{F}_{2}\right)$ is injective.

Theorem 4.5. $\nu_{2}\left(D_{2 i-1}\right)=1+\nu_{2}(2 i)$.

Proof: As in the proof of Theorem 4.3 we have

$$
\begin{gathered}
1-(-5)^{j+1}: P\left(H_{\text {free }}^{2 j}\left(\Omega^{\infty} S^{\infty}\left(\mathbb{C} P_{+}^{\infty}\right)\right)\right) \otimes \mathbb{Z}_{2} \stackrel{(\omega \circ \alpha)^{*}}{\longrightarrow} P\left(H_{\text {free }}^{2 j}\left(B \Gamma_{\infty}^{+}\right)\right) \otimes \mathbb{Z}_{2} \\
\stackrel{\mu_{2}^{*}}{\longrightarrow} H^{2 j}\left(\mathbb{C} P^{\infty} ; \mathbb{Z}_{2}\right) .
\end{gathered}
$$

All groups are copies of $\mathbb{Z}_{2}, \kappa_{j}=D_{j} \tau_{j}$ with $\tau_{j}$ a generator and $\kappa_{j}=(\omega \circ \alpha)^{*}\left(s_{j}\right)$, where $s_{j}$ is the generator of the left term. Suppose that $\nu_{2}\left(D_{j}\right)=2+\nu_{2}(j+1)$ for some $j=2 i-1$. Then $\mu_{2}^{*}\left(\tau_{j}\right)$ would be a generator and dually

$$
\mu_{2 *}: H_{2 j}\left(\mathbb{C} P^{\infty} ; \mathbb{F}_{2}\right) \longrightarrow H_{2 j}^{\text {free }}\left(B \Gamma_{\infty}^{+}\right) \otimes \mathbb{F}_{2}
$$

and hence

$$
\mu_{2 *}: H_{2 j}\left(\mathbb{C} P^{\infty} ; \mathbb{F}_{2}\right) \longrightarrow H_{2 j}\left(B \Gamma_{\infty}^{+}\right) \otimes \mathbb{F}_{2}
$$

would be non-zero. Now apply Theorem 2.4 and Theorem 4.4 to conclude that

$$
H_{4 i-2}\left(\mathbb{C} P^{\infty} ; \mathbb{F}_{2}\right) \stackrel{\left(\omega \circ \alpha \circ \mu_{2}\right)_{*}}{\longrightarrow} H_{4 i-2}\left(\Omega_{0}^{\infty} S^{\infty}\left(\mathbb{C} P_{+}^{\infty}\right) ; \mathbb{F}_{2}\right)
$$

would be non-zero. But this leads to a contradiction as we now argue.

Indeed, by Theorem 2.2 the above map $\left(\omega \circ \alpha \circ \mu_{2}\right)_{*}$ is $\left(1+5 \psi^{-5}, 0\right)_{*}$. The selfmap $\psi^{-5}$ of $\mathbb{C} P^{\infty}$ inducesthe identity on $H_{*}\left(\mathbb{C} P^{\infty} ; \mathbb{F}_{2}\right)$. Hence $1+5 \psi^{-5}$ induces 
the same map on $\mathbb{F}_{2}$-homology as six times the canonical inclusion map:

$$
6 i: \mathbb{C} P^{\infty} \longrightarrow \Omega^{\infty} S^{\infty}\left(\mathbb{C} P^{\infty}\right) \text {. }
$$

But $(6 i)_{*}=0$ on $\mathbb{F}_{2}$-homology in degrees $4 i-2$, giving the desired contradiction. Indeed, $6 i$ is the composition of $2 i$ and multiplication by 3 in the loop sum sense in $\Omega^{\infty} S^{\infty}\left(\mathbb{C} P^{\infty}\right)$. $2 i$ in turn is the composition of the diagonal map on $\mathbb{C} P^{\infty}$ composed with $i \times i$ and loop sum. The diagonal map sends the generator $a_{j}$ of the $2 j$-th homology group to $\Sigma_{s+t=j} a_{s} \otimes a_{t}$. Loop sum replaces tensor product by Pontryagin product, which here is commutative. Thus in $\mathbb{F}_{2}$-homology for $j$ odd, $2 i$ is zero.

\section{Proof of Theorem 2.2}

We offer two proofs of Theorem 2.2. The first one is in the spirit of [MT] and provides an improvement on the geometric construction of $\mu_{p}$ given there. The second proof was suggested by the referee. It is short, purely homotopy theoretic, but it does not provide any insight in the map $\mu_{p}$ from $\Omega^{\infty} S^{\infty} \mathbb{C} P_{+}^{\infty}$ to $B \Gamma_{\infty}^{+}$. The map $\mu_{p}$, constructed from explicit cyclic ramified covers of $\mathbb{C} P^{1}$, provides an important link between the algebraic approach and the topological approach to the mapping class group.

5.1. A constructive proof. In order to prove that the map $\Omega^{\infty} S^{\infty} \mathbb{C} P^{\infty} \rightarrow$ $\Omega^{\infty} S^{\infty}$ induced by $\omega \circ \alpha \circ \mu_{p}$ is zero after $p$-adic completion, we will have to review the construction of $\mu_{p} . \mu_{p}$ depends on $p$ and the choice of $k$; here $k$ can be any integer which is a unit modulo $p^{n}$ for all $n \geq 1$. We take the opportunity to give a variant of the proof for the main theorem in [MT] from sections 3.1-3.3. For the fact that $\alpha$ is a map of infinite loop spaces we refer to section 2 of [MT].

We first describe Riemann surfaces $\Sigma$ with holomorphic actions of the $q$-th roots of unity $\mu_{q} \subset \mathbb{C}^{\times}$. This gives maps $B \mu_{q} \rightarrow B \operatorname{Diff}(\Sigma)$.

Consider a divisor $D=\sum n_{i} p_{i}$ of $\mathbb{C} P^{1}$ with support $A=\left\{p_{0}, p_{1}, \ldots, p_{k}\right\}, n_{i} \in \mathbb{N}$ and $n_{0}+n_{1}+\cdots+n_{k} \equiv 0(\bmod q)$. Assume for $\operatorname{simplicity}$ that $\operatorname{gcd}\left(q, n_{i}\right)=1$ for $i=0,1, \ldots, k$. Let $\Sigma_{D}$ be the branched cover associated with the Galois extension

$$
\mathbb{C}(z) \hookrightarrow \mathbb{C}(z)[T] /(F(T)), \quad F(T)=T^{q}-\prod_{i=0}^{k}\left(z-p_{i}\right)^{n_{i}}
$$

(see e.g. $\left[\mathrm{F}\right.$, chap. 1.8). The Galois group is the group $\mu_{q}$. The surface $\Sigma_{D}$ has a holomorphic action of $\mu_{q}$ with orbit space $\mathbb{C} P^{1}$. The induced map $\pi: \Sigma_{D} \rightarrow \mathbb{C} P^{1}$ is holomorphic, branched over $A$, and $\pi^{-1}\left(p_{i}\right)$ is a single point for each $i$ (since we assumed $\left.\operatorname{gcd}\left(n_{i}, q\right)=1\right)$.

Thus the $\mu_{q}$ action on $\Sigma_{D}$ is free outside $A$, and $A$ is fixed pointwise by all elements in $\mu_{q}$.

Let $\gamma_{i}$ be a small loop in $\mathbb{C} P^{1}$ around $p_{i}$. The fundamental group $\mathbb{C} P^{1} \backslash A$ is the free group of rank $k$ generated by $\gamma_{0}, \ldots, \gamma_{k}$ with the single relation $\prod \gamma_{i}=1$. The covering $\Sigma_{d} \backslash A \rightarrow \mathbb{C} P^{1} \backslash A$ is classified by the map from $\pi_{1}\left(\mathbb{C} P^{1} \backslash A\right)$ to $\mu_{q}$ that sends $\gamma_{i}$ to $e^{2 \pi i n_{i} / q}$. The complex tangent line $T_{p_{i}} \Sigma_{D}$ at $p_{i}$ is a $\mu_{q}$ representation; $u \in \mu_{q}$ multiplies by $u^{\bar{n}_{i}}$ where $\bar{n}_{i} \in \mathbb{Z} / q$ is the multiplicative inverse of $n_{i}$. 
If $D$ and $D^{\prime}$ are two divisors and $q$ divides their difference $D-D^{\prime}$, then there is a biholomorphic map between $\Sigma_{D}$ and $\Sigma_{D^{\prime}}$ that is equivariant w.r.t. the $\mu_{q}$ action. Thus it is only the class of $D$ in $\tilde{H}_{0}(A ; \mathbb{Z} / q)$ that matters.

Recall from Section 3.2 that given a surface bundle $\pi: E \rightarrow X$ there is a diagram

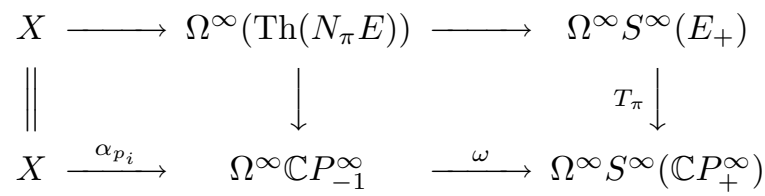

where $\Omega^{\infty}\left(\operatorname{Th}\left(N_{\pi} E\right)\right)=$ colim $\Omega^{n+2}\left(N_{\pi}^{n} e_{n}\right)$ for suitable fat fibrewise embeddings $e_{n}: E \rightarrow X \times \mathbb{R}^{n+2}$. The upper horizontal composition is the Becker-Gottlieb transfer map $t_{E}=t_{\pi}: X \rightarrow \Omega^{\infty} S^{\infty}\left(E_{+}\right)$.

We need the following properties of the Becker-Gottlieb transfer for smooth manifold bundles with compact fiber and compact Lie structure group:

(A1) Let $f: E \rightarrow E^{\prime}$ be a fiberwise homotopy equivalence. Then

$$
t_{E^{\prime}}=\Omega^{\infty} S^{\infty}\left(f_{+}\right) \circ t_{E} \in\left[X, \Omega^{\infty} S^{\infty}\left(E_{+}^{\prime}\right)\right] .
$$

(A2) Suppose

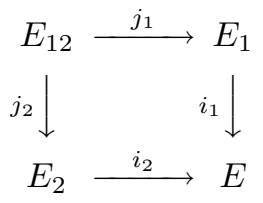

is fiberwise homotopy co-Cartesian. In $\left[X, \Omega^{\infty} S^{\infty}\left(E_{+}\right)\right]$,

$t_{E}=\Omega^{\infty} S^{\infty}\left(i_{1+}\right) \circ t_{E_{1}}+\Omega^{\infty} S^{\infty}\left(i_{2+}\right) \circ t_{E_{2}}-\Omega^{\infty} S^{\infty}\left(i_{12+}\right) \circ t_{E_{12}}$,

where $i_{12}=j_{1} \circ i_{1}=j_{2} \circ i_{2}$.

(A3) If the tangent bundle along fibers $T_{\pi} E$ admits a non-zero section, then $t_{E} \in\left[X, \Omega^{\infty} S^{\infty}\left(E_{+}\right)\right]$is the zero element.

The proof of (A1) and (A2) can be found in [LMS], pp. 189-190 or in [BS]. Property (A3) is much simpler. It follows because

$$
\operatorname{Th}\left(N_{\pi} E\right) \longrightarrow \operatorname{Th}\left(N_{\pi} E \oplus T_{\pi} E\right)
$$

is homotopic to the constant map at $\infty$ whenever $T_{\pi} E$ has an everywhere non-zero section.

Let $\Sigma=\Sigma_{D}$ be the $\mu_{q}$-surface constructed above. We shall study the transfer of the associated smooth surface bundle

$$
\pi: E \mu_{q} \times{ }_{\mu_{q}} \Sigma \longrightarrow B \mu_{q} .
$$

To shorten notation we write

$$
t_{\Sigma}: B \mu_{q} \longrightarrow \Omega^{\infty}\left(E_{+}\right), \quad E=E \mu_{q} \times_{\mu_{q}} \Sigma
$$

for the associated transfer.

Let $D=\sum n_{i} p_{i}$ and $q \in \mathbb{N}$ satisfy $A=\operatorname{supp}(D)=\left\{p_{0}, \ldots, p_{k}\right\}, n_{0}+\cdots+n_{k} \equiv 0$, $\operatorname{gcd}\left(q, n_{i}\right)=1$ for $i=0, \ldots, k$. For each $i$, the inclusion of $E \mu_{q} \times_{\mu_{q}}\left\{p_{i}\right\} \subset E$ induces a map

$$
\hat{p}_{i}: B \mu_{q} \longrightarrow E \longrightarrow \Omega^{\infty} S^{\infty}\left(E_{+}\right) .
$$




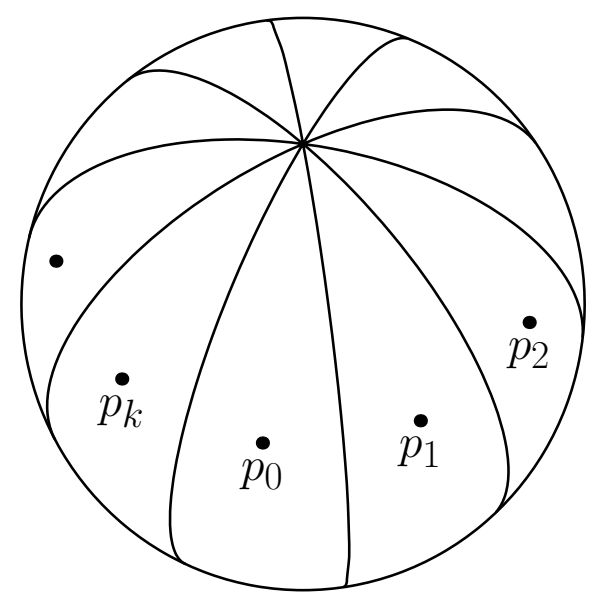

FiguRE 1.

The principal $\mu_{q}$ bundle $E \mu_{q} \rightarrow B \mu_{q}$ induces a transfer from $B \mu_{q}$ to $\Omega^{\infty} S^{\infty}\left(E \mu_{q_{+}}\right)$ $\simeq \Omega^{\infty} S^{\infty}$, and hence

$$
\hat{t}_{q}: B \mu_{q} \longrightarrow \Omega^{\infty} S^{\infty} \longrightarrow \Omega^{\infty} S^{\infty}\left(E_{+}\right)
$$

upon choosing a point of $E$.

Lemma 5.1. The transfer $t_{\Sigma}$ is equal to $\sum \hat{p}_{i}+(1-k) \hat{t}_{q}$ in $\left[B \mu_{q}, \Omega^{\infty} S^{\infty}\left(E_{+}\right)\right]$.

Proof. We make a cell decomposition of $S^{2}=\mathbb{C} P^{1}$ with two 0 -cells $\{0, \infty\}, k+1$ 1-cells $I_{i}$ and $k+12$-cells $D_{i}$ such that $p_{i} \in \operatorname{int} D_{i}$. (See Figure 1.)

There are obvious co-Cartesian diagrams

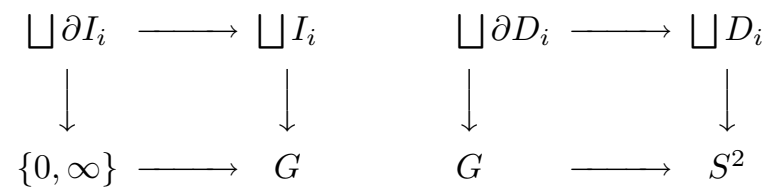

where $G$ denotes the 1-skeleton. This cell structure lifts to a cell structure of $\Sigma$ :
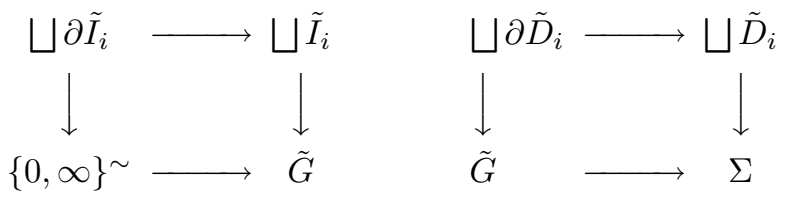

with $\tilde{G}=\pi^{-1}(G)$, etc. We can apply the functor $E \mu_{q} \times{ }_{\mu_{q}}(-)$ to (5.2) and use $(\mathrm{A} 1-\mathrm{A} 3)$ to evaluate $t_{\tilde{G}}$ and $t_{\Sigma}$. First by (A1) and (A2),

$$
t_{\tilde{G}}=(1+k) \hat{t}_{q}+2 \hat{t}_{q}-2(k+1) \hat{t}_{q}=(1-k) \hat{t}_{q},
$$

since $\tilde{I}_{i}=\mu_{q} \times I_{i}, \partial \tilde{I}_{i}=\mu_{q} \times \partial I_{i}$ and $\{0, \infty\}^{\sim}=\mu_{q} \times\{0, \infty\}$. Second, the inclusion of $p_{i}$ in $\tilde{D}_{i}$ is a homotopy equivalence, so $t_{\tilde{D}_{i}}=\hat{p}_{i}$. Moreover, $E \mu_{q} \times_{\mu_{q}} \partial \tilde{D}_{i}=$ $E \mu_{q} \times S^{1}$ has trivial vertical tangent bundle, so $t_{\partial \tilde{D}_{i}}$ is homotopically constant. One moreapplication of (A1-A2) completes the proof. 
The tangent representation $T_{p_{i}} \Sigma$ is given by multiplication with $e^{2 \pi i \bar{n}_{i} / q}$, so an application of Lemma 5.1 gives

Corollary 5.2. The homotopy class of

$$
B \mu_{q} \longrightarrow \Omega^{\infty} \mathbb{C} P_{-1}^{\infty} \longrightarrow \Omega^{\infty} S^{\infty}\left(\mathbb{C} P_{+}^{\infty}\right)
$$

is $\sum \psi^{\bar{n}_{i}}+(1-k) \hat{t}_{q}$. Here $\psi^{\bar{n}_{i}}$ is the composition

$$
\psi^{\bar{n}_{i}}: B \mu_{q} \longrightarrow \mathbb{C} P^{\infty} \longrightarrow \Omega^{\infty} S^{\infty}\left(\mathbb{C} P_{+}^{\infty}\right)
$$

with the left-hand map induced from the group homomorphism $\mu_{q} \rightarrow S^{1}$ that sends $u$ to $u^{\bar{n}_{i}}$.

We are now ready to complete the proof of Theorem 2.2. As in section 3.3 of MT we let $q=p^{n}$ be a prime power and consider the divisor

$$
D=p_{0}+m p_{1}+\cdots+m p_{k}, \quad m \equiv-1 / k\left(\bmod p^{n}\right) .
$$

We use the notation

$$
F(n)=\Sigma_{D}, \quad C_{p^{n}}=\mu_{p^{n}}, \quad \tau_{n}=\hat{t}_{p^{n}}
$$

and consider diagram (5.1) with

$$
X=B \operatorname{Diff}(F(n)), \quad E=E \operatorname{Diff}(F(n)) \times_{\operatorname{Diff}(F(n))} F(n) .
$$

Composing with the map $B C_{p^{n}} \rightarrow B \operatorname{Diff}(F(n))$ induced by the $C_{p^{n}}$ action on $F(n)$, and using that $B \Gamma_{g(n)} \simeq B \operatorname{Diff}(F(n))$ with $g(n)=\frac{1}{2}\left(p^{n}-1\right)(k-1)$ we get the diagram

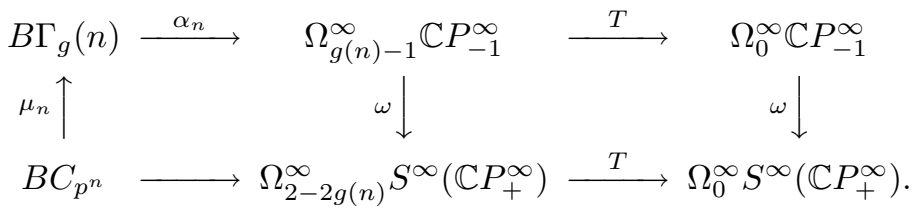

The right-hand horizontal maps are translations of the indicated component into the zero component. The lower horizontal composition is by Corollary 5.2 equal to

$$
\left(1+k \psi^{-k}, \tilde{\tau}_{n}\right) \in\left[B C_{p^{n}}, \Omega^{\infty} S^{\infty}\left(\mathbb{C} P^{\infty}\right) \times \Omega_{0}^{\infty} S^{\infty}\right]
$$

with $\tilde{\tau}_{n}=T \circ \tau_{n}$.

Lemma 5.3. Let $i_{n-1}: B C_{p^{n}-1} \rightarrow B C_{p^{n}}$ be the map associated with $C_{p^{n-1}} \subset C_{p^{n}}$. Then

$$
\left[\tau_{n} \circ i_{n-1}\right]=p\left[\tau_{n-1}\right] \in\left[B C_{p^{n-1}}, \Omega^{\infty} S^{\infty}\right] .
$$

Proof. Let $E$ be a contractible space with a free action of $C_{p^{n}}$ for all $n$, e.g. the union of odd-dimensional spheres $E=\bigcup_{m \geq 1} S^{2 m-1}$. Consider the diagram

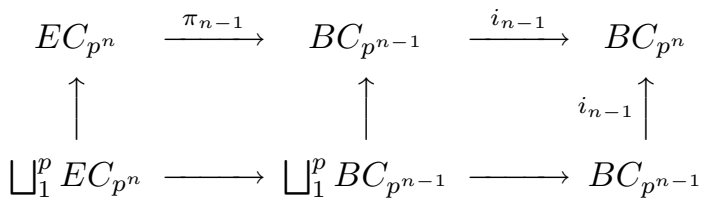

where $B C_{p^{n-1}}$ and $B C_{p^{n}}$ are the orbit spaces $E / C_{p^{n-1}}$ and $E / C_{p^{n}}$ and $i_{n-1}$ is represented by the obvious quotient map. The lower sequence in the above diagram is the pull-back of the upper sequence, and $i_{n-1} \circ \pi_{n-1}=\pi_{n}$. The transfer of a composition is the composition of transfers, and transfers are natural for pull-backs. 
Thus $\left[\tau_{n} \circ i_{n-1}\right]$ is the transfer of the lower sequence composed with the fold map $\bigsqcup_{1}^{p} E \rightarrow E$. This comes out to be $p\left[\tau_{n-1}\right]$.

The Ivanov-Harer stability theorems imply that the map of plus constructions,

$$
B \Gamma_{g(n)-1,2}^{+} \longrightarrow B \Gamma_{g(n)}^{+},
$$

is $b_{n}$-connected with $b_{n}=\left[\frac{g(n)-1}{2}\right]$. Thus

$$
\left[B C_{p^{n}}^{\left(b_{n}\right)}, B \Gamma_{g(n)-1,2}^{+}\right] \simeq\left[B C_{p^{n}}^{\left(b_{n}\right)}, B \Gamma_{g(n)}^{+}\right],
$$

where the superscript indicates the $b_{n}$-skeleton. Let $\mu_{n, 2}: B C_{p^{n}}^{\left(b_{n}\right)} \rightarrow B \Gamma_{g(n)-1,2}^{+}$ correspond to $\mu_{n}$ so that

$$
T \circ \omega \circ \alpha_{n, 2} \circ \mu_{n, 2} \simeq\left(1+k \psi^{-k},(1-k) \tilde{\tau}_{n}\right)
$$

in $\left[B C_{p^{n}}^{\left(b_{n}\right)}, \Omega_{0}^{\infty} S^{\infty}\left(\mathbb{C} P_{+}^{\infty}\right)\right]$. Replacing $\mu_{n, 2}$ by the composition $\mu_{n+l, 2} \circ i_{n+l-1} \circ \cdots \circ i_{n}$ on the left side of (5.3), by Lemma 5.3, the second component on the right-hand side has to be replaced by $p^{l}(1-k) \tilde{\tau}_{n}$. This second component is an element in $\left[B C_{p^{n}}^{\left(b_{n}\right)},\left(\Omega_{0}^{\infty} S^{\infty}\right)_{p}^{\wedge}\right]$ which is a finite $p$-group. Hence, for some $l$ large enough, $p^{l}(1-k) \tilde{\tau}_{n}$ is homotopic to zero.

Consider the subset $G_{n}$ of $\left[B C_{p^{n}}^{\left(b_{n}\right)},\left(B \Gamma_{\infty, 2}^{+}\right)_{p}^{\wedge}\right]$ of elements that satisfy (5.3) with the second component on the right-hand side actually zero. As we have argued it is non-empty. Furthermore, any two elements in $G_{n}$ differ by a map into the fibre of $\omega$. The set $\left[B C_{p^{n}}^{\left(b_{n}\right)}, \Omega_{0}^{\infty} S^{\infty-2}\right]$ is however finite and hence $G_{n}$ is finite. By Tychonov's theorem, the inverse limit of the $G_{n}$ is therefore non-empty. We pick $\tilde{\mu}_{p} \in \lim _{\leftarrow} G_{n}$. Since $\operatorname{colim}_{n \rightarrow \infty} B C_{p^{n}}^{\left(b_{n}\right)}=B C_{p^{\infty}}$ has $p$-adic completion homotopy equivalent to $\left(\mathbb{C} P^{\infty}\right)_{p}^{\wedge}$, the map $\tilde{\mu}_{p}$ extends to a map

$$
\tilde{\mu}_{p}: \Omega^{\infty} S^{\infty}\left(\mathbb{C} P^{\infty}\right) \longrightarrow\left(B \Gamma_{\infty, 2}^{+}\right)_{p}^{\wedge}
$$

and

$$
T \circ \omega \circ \alpha \circ \mu_{p} \simeq\left(1+k \psi^{k}, 0\right) .
$$

This proves that the first column in the matrix described in Theorem 2.2 is $\left(1+k \psi^{-k}, 0\right)$. The second column corresponds to the homotopy class of

$$
\Omega^{\infty} S^{\infty} \stackrel{\mu}{\longrightarrow} \mathbb{Z} \times B \Gamma_{\infty, 2}^{+} \stackrel{\alpha}{\longrightarrow} \Omega^{\infty} \mathbb{C} P_{-1}^{\infty} \stackrel{\omega}{\longrightarrow} \Omega^{\infty} S^{\infty}\left(\mathbb{C} P_{+}^{\infty}\right) .
$$

An infinite loop map with source $\Omega^{\infty} S^{\infty}$ is determined by its restriction to $S^{0}=$ $\{-1,+1\} \hookrightarrow \Omega^{\infty} S^{\infty}$. The $\mu$ above maps the basepoint +1 of $S^{0}$ into $(0, *)$ and the non-basepoint into $(1, *)$. The composition (5.4) maps the basepoint into the basepoint of $\Omega_{0}^{\infty} S^{\infty}\left(\mathbb{C} P^{\infty}\right)$ and the non-basepoint into the basepoint of $\Omega_{-2}^{\infty} S^{\infty}\left(\mathbb{C} P_{+}^{\infty}\right)$. This shows that the second column of the matrix in Theorem 2.2 is $(0,-2)$ as claimed.

5.2. The referee's proof. By Theorem 2.4, we may identify $\mathbb{Z} \times B \Gamma_{\infty}^{+}$with $\Omega^{\infty} \mathbb{C} P_{-1}^{\infty}$ via the map $\alpha$. Hence it suffices to show that the self-map

$$
\left(1+k \psi^{-k},-2\right): \Omega^{\infty} S^{\infty}\left(\mathbb{C} P^{\infty}\right) \times \Omega^{\infty} S^{\infty} \longrightarrow \Omega^{\infty} S^{\infty}\left(\mathbb{C} P^{\infty}\right) \times \Omega^{\infty} S^{\infty}
$$

lifts through

$$
\omega: \Omega^{\infty} \mathbb{C} P_{-1}^{\infty} \rightarrow \Omega^{\infty} S^{\infty}\left(\mathbb{C} P_{+}^{\infty}\right) \simeq \Omega^{\infty} S^{\infty}\left(\mathbb{C} P^{\infty}\right) \times \Omega^{\infty} S^{\infty}
$$

after $p$-completion. By the discussion in section 2.1, the factor of -2 on the summand $\Omega^{\infty} S^{\infty}$ can be lifted by sending the non-basepoint of $S^{0}$ to the basepoint of 
the 1-component in $\Omega^{\infty} \mathbb{C} P_{-1}^{\infty}$, and extending this map to the uniquely determined infinite loop space map from $\Omega^{\infty} S^{\infty}$. Hence, by the fibration sequence $(2.3)$, we are left to show that after $p$-completion the composite map

$$
\Omega^{\infty} S^{\infty}\left(\mathbb{C} P^{\infty}\right) \stackrel{1+k \psi^{k}}{\longrightarrow} \Omega^{\infty} S^{\infty}\left(\mathbb{C} P^{\infty}\right) \stackrel{\partial}{\longrightarrow} \Omega^{\infty} S^{\infty-1}
$$

is null-homotopic.

We recall that $\partial$ is the $S^{1}$-transfer map; cf. [R. Stefan Stolz gave the following, alternative description of the $S^{1}$-transfer map. Let $l: \mathbb{C} P^{\infty} \rightarrow B U$ be the map that represents the reduced canonical line bundle, $\beta: B U \rightarrow \Omega U$ be the Bott map, and $J: U \rightarrow \Omega^{\infty} S^{\infty}$ be the $J$-homomorphism that associates to a unitary transformation the induced map of spheres.

Lemma 5.4 ([St]). The map $\partial$ is homotopic to the infinite loop space map induced by the composite map

$$
\mathbb{C} P^{\infty} \stackrel{l}{\longrightarrow} B U \stackrel{\beta}{\longrightarrow} \Omega U \stackrel{\Omega J}{\longrightarrow} \Omega^{\infty} S^{\infty-1} .
$$

The maps $\psi^{-k}$ give rise to the Adams operations $\psi^{-k}: B U \rightarrow B U$. These operations are stable in the sense that they satisfy the identity

$$
\Omega^{2}\left(\psi^{-k}\right) \circ \beta \simeq \beta \circ\left(-k \psi^{-k}\right) .
$$

Here we identify $U \simeq \Omega B U$. Hence, we have a commutative diagram

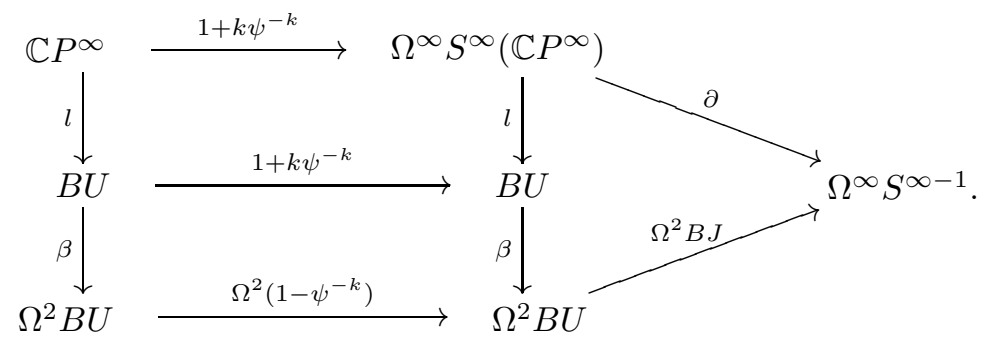

By the affirmed Adams conjecture, after inverting $-k$ and hence after $p$-completion, $B J \circ\left(1-\psi^{-k}\right)$ is null-homotopic. Thus the bottom row defines a null-homotopic map after $p$-completion, which proves (5.5).

\section{Proof of Theorem 1.5}

It follows from [MS, Theorem 7.8] that for odd primes $p$ there is a splitting of $p$-localized spaces

$$
\left(\Omega_{0}^{\infty} \mathbb{C} P_{-1}^{\infty}\right)_{(p)} \simeq T_{(p)} \times B U_{(p)},
$$

where $T_{(p)}$ has torsion homotopy and homology groups. Therefore we have

$$
H_{\text {free }}^{*}\left(\Omega_{0}^{\infty} \mathbb{C} P_{-1}^{\infty} ; \mathbb{Z}_{(p)}\right) \simeq H_{\text {free }}^{*}\left(B U ; \mathbb{Z}_{(p)}\right)=H^{*}\left(B U ; \mathbb{Z}_{(p)}\right) .
$$

For $p=2$ we use the fact that $H_{*}\left(\Omega^{\infty} \mathbb{C} P_{-1}^{\infty} ; \mathbb{F}_{2}\right)$ is a sub-Hopf-algebra of $H_{*}\left(\Omega^{\infty} S^{\infty} \mathbb{C} P_{+}^{\infty} ; \mathbb{F}_{2}\right)$ by theorem 1.3 of [G1]. We need explicit additive generators in low dimensions. Let $a_{i} \in H_{2 i}\left(\Omega^{\infty} S_{1}^{\infty} \mathbb{C} P_{+}^{\infty} ; \mathbb{F}_{2}\right)$ be the non-zero element in the image of $\mathbb{C} P^{\infty} \rightarrow \Omega^{\infty} S_{1}^{\infty} \mathbb{C} P_{+}^{\infty}$ into the 1-component of $\Omega^{\infty} S^{\infty} \mathbb{C} P_{+}^{\infty}$ and let

$$
Q^{s}: H_{*}\left(\Omega^{\infty} S^{\infty} \mathbb{C} P_{+}^{\infty} ; \mathbb{F}_{2}\right) \rightarrow H_{*+s}\left(\Omega^{\infty} S^{\infty} \mathbb{C} P_{+}^{\infty} ; \mathbb{F}_{2}\right)
$$


be the $s$-th homology operation for infinite loop spaces. The following elements form an $\mathbb{F}_{2}$-basis for $H_{*}\left(\Omega_{0}^{\infty} \mathbb{C} P_{-1}^{\infty} ; \mathbb{F}_{2}\right)$ :

$$
\begin{aligned}
& x_{2}=\left(Q^{1} a_{0}\right)^{2} a_{0}^{-4}, \\
& x_{3}=\left(Q^{3} a_{0}\right) a_{0}^{-2}+\left(Q^{2} Q^{1} a_{0}\right) a_{0}^{-4}+\left(Q^{2} a_{0}\right)\left(Q^{1} a_{0}\right) a_{0}^{-4}+\left(Q^{1} a_{0}\right)^{3} a_{0}^{-6}, \\
& x_{4}=\left(a_{1}\right)^{2} a_{0}^{-2}, \quad y_{4}=\left(Q^{2} a_{0}\right)^{2} a_{0}^{-4}, \quad x_{2}^{2},
\end{aligned}
$$

in positive degrees less than 5 . The rational homology of $\Omega_{0}^{\infty} \mathbb{C} P_{-1}^{\infty}$ and $B U$ agree, so

$$
H_{2}^{\text {free }}\left(\Omega_{0}^{\infty} \mathbb{C} P_{-1}^{\infty}\right)=\mathbb{Z}, H_{3}^{\text {free }}\left(\Omega_{0}^{\infty} \mathbb{C} P_{-1}^{\infty}\right)=0, H_{4}^{\text {free }}\left(\Omega_{0}^{\infty} \mathbb{C} P_{-1}^{\infty}\right)=\mathbb{Z} \oplus \mathbb{Z} .
$$

We now employ the Bockstein spectral sequence. For an infinite loop space $X$, it is a singly graded spectral sequence of Hopf algebras with

$$
E_{*}^{1}=H_{*}\left(X ; \mathbb{F}_{2}\right), \quad E_{*}^{\infty}=H_{*}^{\text {free }}(X) \otimes \mathbb{F}_{2} .
$$

The differential $d^{1}$ is the standard Bockstein $[\mathrm{Br}$. The elements listed above for $X=\Omega_{0}^{\infty} \mathbb{C} P_{-1}^{\infty}$ all survive to $E_{*}^{2}$. The general formula for $d^{2}$ applied to an even square [M, Proposition 1.5] gives

$$
d^{2} y_{4}=x_{3}+\left(Q^{3} a_{0}\right) a_{0}^{-2}+\left(Q^{1} a_{0}\right)^{3} a_{0}^{-6}=x_{3}
$$

in $E_{*}^{2}\left(\Omega^{\infty} S_{0}^{\infty} \mathbb{C} P_{+}^{\infty}\right)$, and $d^{2} x_{4}=d^{2} x_{2}^{2}=0$. On the other hand, the $E_{*}^{\infty}$-term is $\mathbb{F}_{2}$, 0 , and $\mathbb{F}_{2} \oplus \mathbb{F}_{2}$ in degrees 2,3 and 4 , respectively. It follows that

$$
E_{2}^{\infty}\left(\Omega_{0}^{\infty} \mathbb{C} P_{-1}^{\infty}\right)=\mathbb{F}_{2}\left\langle x_{2}\right\rangle, \quad E_{4}^{\infty}\left(\Omega_{0}^{\infty} \mathbb{C} P_{-1}^{\infty}\right)=\mathbb{F}_{2}\left\langle x_{4}\right\rangle \oplus \mathbb{F}_{2}\left\langle x_{2}^{2}\right\rangle .
$$

Both $x_{2}^{2}$ and $x_{4}$ are primitive elements (since that is the case in $\Omega^{\infty} S_{0}^{\infty} \mathbb{C} P_{+}^{\infty}$ ), so the reduced diagonal from $E_{4}^{\infty}$ to $E_{2}^{\infty} \otimes E_{2}^{\infty}$ is zero. Dually the squaring map is zero on $H_{\text {free }}^{2}\left(\Omega_{0}^{\infty} \mathbb{C} P_{-1}^{\infty}\right) \otimes \mathbb{F}_{2}$, so $H_{\text {free }}^{*}\left(\Omega_{0}^{\infty} \mathbb{C} P_{-1}^{\infty} ; \mathbb{Z}_{(2)}\right)$ is not polynomial.

Remark. The non-zero differential $d^{2}: E_{4}^{2} \rightarrow E_{3}^{2}$ proves that the two-torsion in $H_{3}\left(B \Gamma_{\infty}\right)$ is $\mathbb{Z} / 4 \mathbb{Z}$.

\section{REFERENCES}

[A1] J.F. Adams, Lectures on generalized cohomology, category theory, homology theory and their applications III, LNM vol. 99, Springer, 1969. MR0251716 (40:4943)

[A2] J.F. Adams, On the groups $J(X)$ II, Topology, vol. 3, 1965, pp. 137-171. MR0198468 (33:6626)

[Ak] T. Akita, Nilpotency and triviality of mod p Morita-Mumford classes of mapping class groups of surfaces, Nagoya Math. J., vol. 165, 2002, pp. 1-22. MR1892095|(2002k:55033)

[BS] J.C. Becker, R.E. Schultz, Axioms for transfers and traces, Math. Z., vol. 227, 1988, pp. 583-605. MR 1621939 (99f:55021)

[B] J. Berrick, An approach to algebraic K-theory, Research Notes in Mathematics, vol. 56, Pitman (Advanced Publishing Program), Boston, 1982. MR0649409 (84g:18028)

[BK] A.K. Bousfield, D.M. Kan, Homotopy Limits, Completions and Localizations, LNM vol. 304, Springer LNM, 1972. MR0365573 (51:1825)

[Br] W. Browder, Torsion in H-spaces, vol. 74, Ann. of Math., 1961, pp. 24-51. MR0124891 (23:A2201)

[C] M. Crabb, $\mathbb{Z} / 2$-Homotopy Theory, , LMS Lecture Note Series, vol. 44, Cambridge University Press, 1980. MR0591680 (83m:55010)

[EE] C.J. Earle, J. Eells, A fibre bundle description of Teichmüller theory, J. Diff. Geom., vol. 3, 1969, pp. 19-43. MR0276999(43:2737a)

[ES] C.J. Earle, A. Schatz, Teichmüller theory for surfaces with boundary, J. Diff. Geom., vol. 4, 1970, pp. 169-185. MR0277000 (43:2737b)

[F] O. Forster, Lectures on Riemann surfaces, Graduate Texts in Mathematics, vol. 81, Springer, New York-Berlin, 1991. MR:1185074 (93h:30061) 
[G1] S. Galatius, Mod p homology of the stable mapping class group, Topology, vol. 43, 2004, pp. 1105-1132. MR2079997 (2006a:57020)

[G2] S. Galatius, Secondary characteristic classes of surface bundles, preprint, math.AT/ 0402226.

[H1] J.L. Harer, The second homology group of the mapping class group of an orientable surface, Invent. Math., vol. 72, 1983, pp. 221-239. MR0700769 (84g:57006)

[H2] J.L. Harer, Stability of the homology of the mapping class groups of orientable surfaces, Annals Math., vol. 121, 1985, pp. 215-249. MR0786348 (87f:57009)

[I] N.V. Ivanov, Stabilization of the homology of Teichmüller modular groups, Original: Algebra i Analiz, vol. 1, 1990, pp. 675-691, Leningrad Math. J. MR:1015128 (91g:57010)

[LMS] G. Lewis, J. P. May, M. Steinberger, Equivariant stable homotopy theory, LNM vol. 1213, Springer, 1986. MR0866482 (88e:55002)

[M] I. Madsen, Higher Torsion in SG and BSG, vol. 143, Math. Z., 1975, pp. 55-80. MR0375307 (51:11503)

[MS] I. Madsen, C. Schlichtkrull, The circle transfer and K-theory, AMS Contemporary Math., vol. 258, 2000, pp. 307-328. MR1778114 (2001e:55021)

[MT] I. Madsen, U. Tillmann, The stable mapping class group and $Q\left(\mathbb{C} P^{\infty}\right)$, vol. 145 , Invent. Math., 2001, pp. 509-544. MR1856399 (2002h:55011)

[MSt] J. Milnor, J. Stasheff, Characteristic Classes, Study, vol. 76, Princeton University Press, 1974. MR0440554 (55:13428)

[MW] I. Madsen, M. Weiss, The stable moduli space of Riemann surfaces: Mumford's conjecture, math.AT/0212321.

[Mi] E.Y. Miller, The homology of the mapping class group, vol. 24, J. Diff. Geom., 1986, pp. 1-14. MR0857372 (88b:32051)

[Mo] S. Morita, Characteristic classes of surface bundles, vol. 90, Invent. Math., 1987, pp. 551577. MR0914849 (89e:57022)

$[\mathrm{Mu}] \quad \mathrm{D}$. Mumford, Towards an enumerative geometry of the moduli space of curves in Arithmetic and Geometry (M. Artin and J. Tate, editors), Progr. Math., vol. 36, Birkhäuser, 1983, pp. 271-328. MR0717614 (85j:14046)

[R] D. Ravenel, The Segal conjecture for cyclic groups and its consequences, vol. 106, Amer. J. Math., 1984, pp. 415-446. MR0737779 (85g:55015)

[S] G. Segal, The stable homotopy of complex projective space, vol. 24, Quart. J. Math. Oxford, 1973, pp. 1-5. MR0319183 (47:7729)

[St] S. Stolz, Relationships between transfer and J-homomorphisms, vol. 125, Bonner Mathematische Zeitschriften, Universität Bonn, 1980. MR0598816 (82g:57012)

[T] U. Tillmann, On the homotopy of the stable mapping class group, vol. 130, Invent. Math., 1997, pp. 257-275. MR.1474157 (99k:57036)

Department of Mathematics, Stanford University, Stanford, California 94305

E-mail address: galatius@math.stanford.edu

Matematisk Institut, Aarhus Universitet, 8000 Aarhus C, Denmark

E-mail address: imadsen@imf.au.dk

Mathematical Institute, 24-29 St. Giles Street, Oxford OX1 3LB, United Kingdom

E-mail address: tillmann@maths.ox.ac.uk 